\title{
Extending general covariance: Moyal-type noncommutative manifolds
}

\author{
Martin Bojowald, ${ }^{1, *}$ Suddhasattwa Brahma, ${ }^{2,3, \dagger}$ Umut Buyukcam, ${ }^{1, \$}$ and Michele Ronco ${ }^{1,4,5,8}$ \\ ${ }^{1}$ Institute for Gravitation and the Cosmos, The Pennsylvania State University, 104 Davey Lab, \\ University Park, Pennsylvania 16802, USA \\ ${ }^{2}$ Center for Field Theory and Particle Physics, Fudan University, 200433 Shanghai, China \\ ${ }^{3}$ Asia Pacific Center for Theoretical Physics, Pohang 37673, Republic of Korea \\ ${ }^{4}$ Dipartimento di Fisica, Università di Roma "La Sapienza”, P.le A. Moro 2, 00185 Roma, Italy \\ ${ }^{5}$ INFN, Sez. Romal, P.le A. Moro 2, 00185 Roma, Italy
}

(Received 13 June 2018; published 30 July 2018)

\begin{abstract}
In the Hamiltonian formulation of general relativity, Einstein's equation is replaced by a set of four constraints. Classically, the constraints can be identified with the generators of the hypersurfacedeformation Lie algebroid (HDA) that belongs to the groupoid of finite evolutions in space-time. Taken over to deformed general relativity, this connection allows one to study possible Drinfeld twists of spacetime diffeomorphisms with Hopf-algebra techniques. After a review of noncommutative differential structures, two cases-twisted diffeomorphisms with standard action and deformed (or $\star-$ ) diffeomorphisms with deformed action-are considered in this paper. The HDA of twisted diffeomorphisms agrees with the classical one, while the HDA obtained from deformed diffeomorphisms is modified due to the explicit presence of $\star$-products in the brackets. The results allow one to distinguish between twisted and deformed symmetries, and they indicate that the latter should be regarded as the relevant symmetry transformations for noncommutative manifolds. The algebroid brackets maintain the same general structure regardless of space-time noncommutativity, but they still show important consequences of nonlocality.
\end{abstract}

DOI: 10.1103/PhysRevD.98.026031

\section{INTRODUCTION}

Thanks to general relativity (GR), gravitational interactions are understood as purely geometric phenomena which can be described in terms of a metric, an affine connection, and a curvature defined on a (pseudo-) Riemannian manifold. The symmetry of general covariance is an important governing principle which determines possible dynamical theories. Accordingly, one may attempt to quantize gravity by analyzing possible quantum spacetime symmetries which determine the structure of the geometry of the system. As shown in Refs. [1-14], the concept of absolutely sharp points, one of the cornerstones of Riemannian geometry, should then be expected to break down.

A general mathematical structure that can make sense of such a space-time is provided by noncommutative

\footnotetext{
*bojowald@gravity.psu.edu

†suddhasattwa.brahma@gmail.com

*uxb101@psu.edu

\$michele.ronco@roma1.infn.it
}

Published by the American Physical Society under the terms of the Creative Commons Attribution 4.0 International license. Further distribution of this work must maintain attribution to the author(s) and the published article's title, journal citation, and DOI. Funded by SCOAP. geometry [15-22]. The approach pioneered by Connes starts by recognizing the tight relation between the geometrical properties of space and the algebra of continuous functions on it. For commutative algebras, the theorem of Gelfand and Neimark guarantees that there is an equivalence between compact Hausdorff spaces and $\mathrm{C}^{*}$-algebras. The main idea is then to extend this equivalence to noncommutative algebras. Although one cannot reconstruct the space from a noncommutative algebra, it is still possible to introduce generalized versions of the metric and a related differential calculus by means of the Dirac operator acting on functions of the algebra [23-25].

A different implementation of noncommutative geometry involves a notion of deformed symmetries often referred to as quantum groups [26,27]. For more than twenty years now, the study of possible quantum deformations of relativistic symmetries has been intensely pursued [28-36], and supersymmetric extensions have been considered as well [37-39]. The present understanding is that, even with noncommutativity, it is still possible to have a ten-dimensional local symmetry group (replacing classical Poincaré transformations) by means of what is known as a "Drinfeld twist" [40-42].

In the case of flat space-time, twists allow one to interpret noncommutative versions of Minkowski spacetime as objects which are, in a certain sense, dual to suitable 
deformations of the Poincare algebra. Identifying the dimensionful deformation parameter $\lambda$ (or $\kappa \sim 1 / \lambda$ ) with the Planck length $\ell_{\mathrm{Pl}}=\sqrt{\hbar G / c^{3}}$ (Planck mass $m_{\mathrm{Pl}}=$ $\sqrt{c \hbar / G})$, these models provide a mathematical realization of the proposal of doubly (or deformed) special relativity $[43,44]$, which argues that Planck-scale effects should necessitate a description of space-time physics in terms of two relativistic invariants: $\lambda$ or $\kappa$ in addition to the speed of light $c$. In spite of this success, the extension of noncommutativity to curved manifolds remains an open issue, which is of particular importance because one of the main applications of quantum groups and the associated space-time noncommutativity is the characterization of Planck-scale physics. They should therefore have the potential to be a candidate theory of quantum gravity (QG), which has to include curved space-time solutions.

Attempts to quantize 3-dimensional gravity have met with more success [45-54], but much work remains to be done to generalize these results to the 4-dimensional theory of physical interest. Understanding how to quantize GR or, even more generally, the class of all possible covariant theories remains center stage in the research program of noncommutative geometry and the associated deformation of gauge groups. Another important stimulus to study the deformation of diffeomorphisms groups, which can be regarded as the gauge symmetries of GR, comes from string theory. In this context, it has been shown that coordinates obey canonical noncommutativity if a background tensor field (or $B$-field in short) is present [55-59].

In the last two decades, the study of Hopf algebras from a physical perspective has given rise to a rather sizable literature on quantum Minkowski spacetimes [60-63]. These zero-curvature models are often considered toy models for the flat limit of a (still to be found) QG theory. In some very rare cases, they have even proved useful for phenomenological proposals [64]. The main idea is to promote coordinates $x^{\mu}$ to noncommuting operators $\hat{X}^{\mu}$ with non-trivial commutators of the form $\left[\hat{X}^{\mu}, \hat{X}^{\nu}\right]=$ $i \theta^{\mu \nu}(\hat{X})=i \theta^{\mu \nu}+i \Theta_{\rho}^{\mu \nu} \hat{X}^{\rho}$. Thanks to Weyl-Moyal maps, which had been first introduced to study the phase space of quantum mechanics, one can trade operator-valued coordinates for functions living on a classical manifold but equipped with a nonstandard multiplication rule. This procedure introduces a noncommutative $\star$-product, whose main feature is nonlocality. Such quantum deformations of coordinate spaces based on algebraic relations have been extensively studied since the seminal paper by Snyder [65]. The best known examples are given by $\theta$ (or Moyal) canonical space [66], $\kappa$-Minkowski spacetime invariant under the $\kappa$-Poincaré algebra [67,68], $q$-deformations of Lie groups [69-71], and the fuzzy sphere [20,72].

All this literature mainly focused on the construction of noncommutative Minkowski space-times but did not contemplate extensions to curved versions. Some progress has been made in the quantization of symmetry-reduced
GR solutions such as de Sitter [73], anti-deSitter [74], and Friedmann-Robertson-Walker [75] backgrounds. Nevertheless, the situation for the quantization of the full group of diffeomorphisms remains unclear and the relevant literature is fragmented. The main obstacle seems to be the proper definition of coordinate transformations and a self-consistent calculus once coordinates have been promoted to noncommuting objects. It is not difficult to realize that noncommutativity introduces a preferred frame (or coordinate choice) and thus is not compatible with the standard symmetries. For instance, if we assume that $\left[\hat{X}_{\rho}, \hat{X}_{\sigma}\right]=i \theta_{\rho \sigma}$, as it is the case for the canonical or Moyal-Weyl noncommutative spacetime, then the transformed coordinates $\widehat{X}_{\mu}^{\prime}=\hat{X}_{\mu}+\hat{\xi}_{\mu}$, with a vector field $\hat{\xi}_{\mu}$ depending linearly on $\hat{X}_{\mu}$ (as required for rotations and boosts), do not obey the original commutation relation $\left[\widehat{X}_{\rho}^{\prime}, \widehat{X}_{\sigma}^{\prime}\right] \neq i \theta_{\rho \sigma}$. To avoid this, as we briefly hinted above, one needs to quantize (or deform) the symmetry group in a specific way. Such a deformation theory in complete form is not available for diffeomorphism groups. For this reason, we do not yet have a widely accepted noncommutative theory of gravity.

A possible way out, proposed in Ref. [76], lies in restricting the group of diffeomorphisms to those transformations that preserve coordinate noncommutativity. It has been recognized [76] that, in the case of canonical space, this proposal corresponds to a restriction to volumepreserving diffeomorphisms. One therefore obtains a connection with unimodular gravity [77,78]. Another possibility, explored in Ref. [79], is a generalization of the Seiberg-Witten map [55] to GR by gauging the Lorentz group. A drawback of such an approach is that it forces one to use a complex metric structure [79]. An alternative perspective on the interplay between gravity and noncommutative geometry is offered for instance by Refs. [80,81], where matrix models of Yang-Mills type are developed. In this case (see [81] for a review), there is no space-time manifold and space-time coordinates are replaced by a set of (noncommutative) matrices used to embed different geometries depending on their algebra. The notion of Riemannian geometry should then emerge from a path integral over matrix configurations corresponding to different topologies. The most notable example of quantum geometry realized in terms of a matrix model is given by the fuzzy sphere [20,82-84]. A recent approach using twisted bundles has been developed in $[85,86]$.

Perhaps one of the most promising paths proposed so far is that of twisted diffeomorphisms $[87,88]$. The main idea is to replace the diffeomorphism invariance of GR by its twisted version. This is done by deforming the Hopf algebra structure of the universal enveloping algebra of the Lie algebra of vector fields by twisting the coproduct by means of Drinfeld twists $[87,88]$. The action of diffeomorphisms on single fields then stays unmodified while the Leibniz rule (which provides the action on two or more 
fields) is changed. As a result, the $\star$-product of two (or more) fields is covariant under twisted diffeomorphisms. Finally, one can write down a modification of the EinsteinHilbert action which is invariant under twisted diffeomorphisms thanks to an appropriate $\star$-product. Given the potential of such an approach, Ref. [89] explored whether such a (twisted) noncommutative gravity can be obtained from closed strings with an external $B$-field in the Seiberg-Witten limit. Unfortunately, there has been no way of matching this limit of string theory with the gravity model of Ref. [87]. Moreover, as already pointed out in Refs. [89,90], we stress that twisted symmetries are not genuine deformations of classical symmetries but rather mappings of the classical symmetries on spaces with noncommutative $\star$-products. Following what has been done for other gauge groups [17-19], one should properly deform also the action on single fields in order to have a definition of $\star$ (or deformed) diffeomorphisms. To our knowledge, no such formulation is currently available in the literature. The introduction of deformed diffeomorphisms, as opposed to twisted diffeomorphisms, represents one of the main objectives of the present work.

In addition, we propose a new line of inquiry and ask whether diffeomorphisms can be consistently quantized in the sense of a deformation theory in analogy to what has been already done for the special relativistic (SR) group of Poincaré symmetries. We therefore provide candidate structures for any deformed general relativistic theory, without using specific actions or dynamical equations. In contrast to most previous studies of noncommutative geometry, we follow a canonical approach. Along the lines of the classical analysis of Dirac [91] and Arnowitt-DeserMisner (ADM) [92], it should be possible to perform a $3+1$-splitting of the action of Ref. [87]. Poisson brackets of the resulting scalar and momentum constraints would then lead to the corresponding hypersurface-deformation algebroid (HDA) or Dirac spacetime algebra [91,93,94]. Unfortunately, however, the full ADM machinery turns out to be rather involved when it is applied to gravitational actions on noncommutative manifolds.

As we point out in this paper, there is a shortcut that can provide us with general (that is, action-independent) hints for hypersurface deformations or diffeomorphisms on such manifolds. It therefore leads us to a notion of deformed general covariance. The shortcut is motivated by recent results of Ref. [95] for classical smooth manifolds, further developed in Ref. [96] under weaker assumptions that allow one to bring in some quantum-gravity effects. For our purposes here, the main achievement is the recognition that the symmetry structure of hypersurface deformations (which is usually described as a "Lie algebra with structure functions" in the physics literature) is that of a Lie algebroid which can be derived from a groupoid of finite evolutions between spacelike hypersurfaces in Lorentzian manifolds. (A similar Euclidean version also exists.)
In particular, the rather complicated Poisson brackets between the gravitational constraints of canonical gravity are reproduced by the tangential and normal components of Lie brackets between suitable (Gaussian) space-time vector fields. In order to inspect the HDA for noncommutative spacetimes, it is then not necessary to know the explicit expressions of constraints as phase-space functions, which in fact would not be available for noncommutative gravity. It is sufficient to introduce a suitable differential calculus and apply it to such a noncommutative version of a tangential-normal decomposition by following the steps of recent analyses [95,96], observing certain consistency conditions extracted from [95].

We will start by modifying general coordinate transformations of commuting variables into diffeomorphisms of noncommuting functions. Moyal-Weyl maps allow us to treat operator-valued objects as standard functions, but multiplied with a noncommutative $\star$-product. (That is, to introduce noncommutativity we do not need to change the classical function space, but only the product in the algebra of functions.) At the classical level, infinitesimal diffeomorphisms form a Lie algebra with an extension of their action from vector fields to tensor fields because the standard Leibniz rule applies. We deform this structure by using Drinfeld twists and, thus, define a deformed differential geometry. When we analyze the case of twisted diffeomorphisms, the algebra remains unchanged while the comultiplication changes, confirming the suggestions made in Refs. [87,88]. Twisted diffeomorphisms are opposed to deformed (or $\star-$ ) diffeomorphisms which we introduce and discuss for the first time.

In the definition of the action on single fields we follow established results in the literature, while we explore two possibilities regarding the comultiplication rule or coalgebra sector of $\star$-diffeomorphisms. We will first try to mimic the situation of $U(N)$ noncommutative gauge theories $[17,19,21,97-101]$ and work with trivial coproducts. (The standard Leibniz rule then applies.) We will note several drawbacks of retaining the standard Leibniz rule, which leads us to propose a suitable deformation of comultiplication. In both cases we are able to compute the HDA brackets and show that, as opposed to the twisted case, there are $\star$ product deformations in the algebra which distinguish deformed from twisted diffeomorphisms. Sharing the concerns raised in Ref. [89], we expect that $\star$-diffeomorphisms, rather than twisted ones, should be chosen as the symmetries of a noncommutative theory of gravitation.

Together with previously established results in the literature on noncommutative gravity, our work provides general results about possible formulations of a deformed gravity theory, defined with a deformed differential geometry on noncommutative hypersurfaces. The closed brackets of hypersurface-deformation generators with star products found here can be used to test the covariance of existing proposals for noncommutative gravity theories, 
but they may also prove useful in the construction of new such models or in a classification of all possible deformations of classically covariant theories.

To some extent, noncommutative gravity represents an independent approach to QG. However, we wish to stress that, besides the aforementioned seminal papers [55-57] showing the appearance of noncommutativity in string theory due to the presence of external fields, additional support to a possible role for spacetime noncommutativity in string theory has been recently claimed in Refs. [102-104]: There, it has been shown that the target space of closed strings is noncommutative regardless of the specific features of the background. Additional motivation for our work comes from the recent interest in modifications and/or generalizations of the HDA found in the QG literature [105-113], including a possible way to ascribe Minkowski spacetime quantization and Poincaré symmetry deformation to loop quantum gravity corrections [114-121]. General deformations of the HDA have also been studied recently in Ref. [122], where the authors found a (partial) no-go theorem forbidding specific modifications of the scalar constraint in a general covariant theory. It is possible to regard our work as an explicit example showing that the assumptions of such a theorem can be weakened so as to evade the original conclusions.

Our paper is organized as follows. In Sec. II, we first review the definitions of Lie algebroids and rederive the classical HDA starting from the Lie brackets of a suitable class of space-time vector fields. Then, we remind the reader of the notions of Hopf algebras and introduce a differential calculus on noncommutative manifolds. Vectors, differential forms, tensors, $\star$-Lie derivatives, inner products, and index contraction are all defined. Two different notions of brackets are introduced-Moyal and $\star$-Lie brackets - together with a discussion of their differences. Section III is dedicated to the analysis of hypersurface deformations generated by twisted diffeomorphisms with the Moyal $\star$-product. After defining a proper modification of the classical condition on space-time vector fields, we compute the Lie brackets between them and then decompose the result into normal and tangential parts, thereby obtaining a twisted version of the HDA. Confirming the expectations of Refs. [87,88], we find that the HDA is unmodified. This result also ensures that twisted gravity possesses the same degrees of freedom (d.o.f.) as classical GR. In Sec. IV, we focus on deformed diffeomorphisms. Two different possibilities for the coalgebra sector are considered before an analysis analogous to the previous case is carried out. The resulting HDA is deformed due to the presence of explicit $\star$-product contributions. Finally, we draw our conclusions and sketch an outlook in Sec. V.

\section{MATHEMATICAL PRELIMINARIES}

The main mathematical tools used here, Lie algebroids and Hopf algebras, are reviewed in this section.

\section{A. Lie algebroids}

We closely follow $[95,96]$ but similar content can also be found, for instance, in [123]. A Lie algebroid is a vector bundle $A$ over a smooth base manifold $B$ together with a Lie bracket $[\cdot, \cdot]_{A}$ on the set $\Gamma(A)$ of sections of $A$ and a bundle map $\rho: \Gamma(A) \rightarrow \Gamma(T B)$, called the anchor, provided that the following two properties are satisfied:

(i) $\rho:\left(\Gamma(A),[\cdot, \cdot]_{A}\right) \rightarrow(\Gamma(T B),[\cdot, \cdot])$ is a Lie-algebra homomorphism: for any $\xi, \eta \in \Gamma(A)$, we have $\rho\left([\xi, \eta]_{A}\right)=[\rho(\xi), \rho(\eta)]$ (the Lie bracket of vector fields in $\Gamma(T B)$ ).

(ii) For any $\xi, \eta \in \Gamma(A)$ and $f \in C^{\infty}(B)$, the Leibniz rule $[\xi, f \eta]_{A}=f[\xi, \eta]_{A}+(\rho(\xi) f) \eta$ holds.

If the base manifold $B$ is a point, the Lie algebroid is a Lie algebra. Let us also mention that, in the case of Lie algebroids, one needs to generalize the notion of Lie algebra morphisms if one desires to identify classes of equivalence. However, morphisms between algebroids will not play any role in our analysis. We refer the interested reader to Ref. [123] and references therein.

We are primarily interested in the specific Lie algebroid of hypersurface-deformations, which provides a mathematical formulation of the Poisson brackets of gravitational constraints [91-93]. Gauge transformations generated by the constraints are equivalent to space-time diffeomorphisms. In a canonical formulation, invariance under these transformations ensures that observables of the theory are independent of the particular embedding of spatial hypersurfaces in space-time. An explicit derivation of hypersurface-deformation brackets can make use of coordinate choices to simplify calculations. The closure of the brackets in the form of a Lie algebroid then ensures that they are covariant under changes of the embedding.

A convenient choice turns out to be given by Gaussian embeddings, which are defined such that the space-time metric $g_{\mu \nu}$ assumes a Gaussian form:

$$
\mathrm{d} s^{2}=-\mathrm{d} t^{2}+q_{a b} \mathrm{~d} x^{a} \mathrm{~d} x^{b} .
$$

Thus, for the components of $g_{\mu \nu}$ one has

$$
g_{\mu \nu}=-n_{\mu} n_{\nu}+q_{a b} X_{\mu}^{a} X_{\nu}^{b}
$$

with the spatial metric $q_{a b}$. We have written the metric in a basis dual to $\left(n^{\mu}, X_{a}^{\mu}\right)$, where $n^{\mu}$ is the unit normal to a family of space-like hypersurfaces $\Sigma_{t}$ (at constant $t$ ), while $X_{a}^{\mu}$ form a basis of $T \Sigma_{t}$. With these conditions, we have the orthonormality relations $g_{\mu \nu} n^{\mu} n^{\nu}=-1$ and $g_{\mu \nu} n^{\mu} X_{a}^{\nu}=0$. Following the ADM treatment of canonical gravity [92], we then decompose the time-evolution vector field $\tau^{\mu}$ by $\tau^{\mu}=N n^{\mu}+M^{a} X_{a}^{\mu}$, where $N$ is the lapse function and $M^{a}$ the shift vector field.

A foliation which is Gaussian for one embedding is, in general, not Gaussian for a different embedding. 
Gaussianity is therefore not preserved by general coordinate transformations. We can, however, restrict the class of transformations to diffeomorphisms generated by Gaussian vector fields $v^{\mu}$ obeying

$$
i_{n} \mathcal{L}_{v} g=0,
$$

or, in components,

$$
n^{\mu} \mathcal{L}_{v} g_{\mu \nu}=0 .
$$

Here (and throughout the paper) $i_{w}$ stands for the internal product (or contraction) with a vector field $w$. The normal components of the metric remain invariant under transformations along the direction of such a $v^{\mu}$, preserving the Gaussian form. Choosing a Gaussian embedding corresponds to fixing a representative in each equivalence class of hypersurface embeddings, in which the subset of Gaussian $v^{\mu}$ furnishes the remaining coordinate freedom.

Expanding the Lie derivative, the Gaussian condition can be rewritten as

$$
n^{\mu} v^{\rho} \partial_{\rho} g_{\mu \nu}+n^{\mu}\left(\partial_{\mu} v^{\rho}\right) g_{\rho \nu}+n^{\mu}\left(\partial_{\nu} v^{\rho}\right) g_{\rho \mu}=0,
$$

resulting in

$$
v^{\rho} \mathrm{d} n_{\rho \nu}+\partial_{\nu}\left(v^{\rho} g_{\rho \mu} n^{\mu}\right)+g_{\mu \nu}[n, v]^{\mu}=0 .
$$

We used the Cartan identity, the definition of the Lie bracket, and $(\mathrm{d} n)_{\mu \nu}=\partial_{\mu} n_{\nu}-\partial_{\nu} n_{\mu}$. Due to the Gaussian from of the metric (2), we have $\mathrm{d} n=0$ because $n=\mathrm{d} t$ is closed. Decomposing the Gaussian vector in the basis chosen above-that is, writing $v^{\mu}=N n^{\mu}+M^{a} X_{a}^{\mu}$-we then have

$$
-\partial_{\nu} N+g_{\mu \nu}\left(n^{\mu} n^{\rho} \partial_{\rho} N+[n, M]^{\mu}\right)=0,
$$

where we have used the orthogonality of the basis. (Although we use the same notation for components $N$ and $M^{a}$ of a Gaussian vector field and the time-evolution vector field, the former are more general since they refer to a coordinate change.) Projecting this expression along normal and tangential directions, respectively, we find

$$
\partial_{\nu} N=0 \quad \text { and } \quad[n, M]^{a}=q^{a b} \partial_{b} N .
$$

Here, $q^{a b}$ is the inverse of the spatial metric. (The bracket $[n, M]^{\mu}$ does not have a normal component thanks to the geodesic property of $n^{\mu}$ for a Gaussian system; see [96] for details.)

We can now compute the HDA by calculating the Lie bracket between two Gaussian vector fields:

$$
\begin{aligned}
{\left[v_{1}, v_{2}\right]^{\mu}=} & v_{1}^{\rho} \partial_{\rho} v_{2}^{\mu}-v_{2}^{\rho} \partial_{\rho} v_{1}^{\mu} \\
= & \left(N_{1} \mathcal{L}_{n} N_{2}-N_{2} \mathcal{L}_{n} N_{1}+\mathcal{L}_{M_{1}} N_{2}-\mathcal{L}_{M_{2}} N_{1}\right) n^{\mu} \\
& +\left[M_{1}, M_{2}\right]^{\mu}+N_{1}\left[n, M_{2}\right]^{\mu}-N_{2}\left[n, M_{1}\right]^{\mu}
\end{aligned}
$$

$$
\begin{aligned}
= & \left(\mathcal{L}_{M_{1}} N_{2}-\mathcal{L}_{M_{2}} N_{1}\right) n^{\mu} \\
& +\left[M_{1}, M_{2}\right]^{\mu}+q^{\mu b}\left(N_{1} \partial_{b} N_{2}-N_{2} \partial_{b} N_{1}\right),
\end{aligned}
$$

where we decomposed both $v_{1}$ and $v_{2}$ in the basis $(n, X)$, and then used the Eq. (8). The terms of the type $\mathcal{L}_{n} N=$ $n^{\rho} \partial_{\rho} N$ are all zero due to the first equality in (8). In order to obtain the HDA, we have to extract normal and tangential contributions: If $N_{1}=N_{2}=0$,

$$
\left[v_{1}, v_{2}\right]^{\mu}=\left[M_{1}, M_{2}\right]^{\mu},
$$

if $M_{1}^{a}=0$ and $N_{2}=0$,

$$
\left[v_{1}, v_{2}\right]^{\mu}=-n^{\mu} \mathcal{L}_{M_{2}} N_{1},
$$

and if $M_{1}^{a}=0=M_{2}^{a}$,

$$
\left[v_{1}, v_{2}\right]^{\mu}=q^{\mu b}\left(N_{1} \partial_{b} N_{2}-N_{2} \partial_{b} N_{1}\right) .
$$

Finally, we view the pairs $\left(N, M^{a}\right)$ as fibers of a Lie algebroid over the space of spatial metrics, and interpret the three cases of $\left[v_{1}, v_{2}\right]^{\mu}$ as Lie-algebroid brackets

$$
\begin{gathered}
{\left[\left(0, M_{1}^{a}\right),\left(0, M_{2}^{b}\right)\right]=\left(0, \mathcal{L}_{M_{1}} M_{2}\right),} \\
{\left[(N, 0),\left(0, M^{a}\right)\right]=\left(-\mathcal{L}_{M} N, 0\right),} \\
{\left[\left(N_{1}, 0\right),\left(N_{2}, 0\right)\right]=\left(0,\left(N_{1} \partial_{b} N_{2}-N_{2} \partial_{b} N_{1}\right) q^{a b}\right) .}
\end{gathered}
$$

(The anchor map is given by the Lie derivative of the metric along $\tau^{\mu}=N n^{\mu}+M^{a} X_{a}^{\mu}$; see [95].) With these brackets, pairs $\left(N, M^{a}\right)$ form the hypersurface-deformation Lie algebroid over the space of spatial metrics. Spatial diffeomorphisms form a subalgebroid which is also a Lie algebra, while the brackets involving only normal deformations depend on the inverse-metric components as coordinates on the base manifold (the "structure functions"). (We note that the base manifold can be extended to the full phase space of general relativity, given by spatial metrics and extrinsic curvature, or linear combinations of the latter components. While this extension is not necessary in the classical algebroid, it may be required for some quantum effects as we will see later in this paper.)

The derivation presented here has several advantages over the usual ones in canonical gravity. It is much shorter and minimizes the amount of technical calculations. Moreover, it utilizes space-time tensor calculus and implements the $3+1$-split only by decomposing vector fields. It is therefore ideal for an application to nonclassical spacetime structures in which some versions of tensor calculus exist. The rest of our work is dedicated to an application of these methods to the deformation theory of this algebroid in order to have a notion of (deformed) general covariance for noncommutative manifolds. We will focus on the brackets 
and not discuss the anchor. As shown in [123], the latter is not subject to deformations.

One question to be discussed in more detail is the definition of Gaussian systems in nonclassical space-times. The Gaussian nature, by itself, is not relevant because it just constitutes a choice of gauge fixing. However, the Gaussian system simplifies the classical derivation, and it makes it easier to check two important consistency conditions which we emphasize here: (i) The derivation of the hypersurfacedeformation brackets requires us to extend the fields $N$ and $M^{a}$ from a given hypersurface into a space-time neighborhood. Only such an extension makes it possible to compute the space-time Lie derivative of two vector fields in (9) and then decompose the result into normal and spatial components. In the classical derivation, such an extension is possible thanks to the form of the differential equation (8), which are well-posed with $N$ and $M^{a}$ as initial conditions on one hypersurface. (ii) The resulting hypersurfacedeformation brackets (17) depend only on spatial data, given by the fields $N$ and $M^{a}$ together with the spatial metric $q_{a b}$. It is therefore possible to interpret them as Liealgebroid relations over the space of metrics. There is no dependence on properties of the embedding of a hypersurface in space-time.

In our new derivations below, we will take a pragmatic approach and look for a generalization of the Gaussian condition such that these two consistency conditions are still satisfied. From this perspective, the main advantage of the Gaussian system turns out to be that it leads to a normal vector $n^{\mu}$ with coordinate-independent components.

\section{B. Hopf algebras and noncommutative calculus}

We now introduce the basic notion of Hopf algebras and the associated noncommutative calculus [87]. We will define only those objects that will be necessary for our analysis.

\section{Hopf algebras}

Let us start by introducing the vector space $\mathbb{K}$ of smooth real or complex vector fields on our classical (commutative) differentiable manifold $\mathcal{M}$. One can always equip $\mathbb{K}$ with a Lie bracket $[u, v]$ which obeys the Jacobi identity. The pair $\mathcal{A}:=(\mathbb{K},[\cdot, \cdot])$ is the Lie algebra of classical infinitesimal diffeomorphisms on $\mathcal{M}$. Infinitesimal transformations of tensors under diffeomorphisms are provided by the Lie derivative $\mathcal{L}_{v}$ which obeys $\mathcal{L}_{v} \circ \mathcal{L}_{u}-\mathcal{L}_{u} \circ \mathcal{L}_{v}=\mathcal{L}_{[v, u]}$ where $\circ$ stands for composition.

The Lie derivative of a tensor produces a tensor of the same type and weight. We shall see in Sec. IV that $\star$-diffeomorphisms obeying the standard Leibniz rule do not satisfy such a condition. We will therefore be led to a suitable modification of comultiplication. Classically, infinitesimal diffeomorphisms act on tensor products of tensor fields, $\tau \otimes \tau^{\prime}$, by means of the Leibniz formula $\mathcal{L}_{v}\left(\tau \otimes \tau^{\prime}\right)=\left(\mathcal{L}_{v} \tau\right) \otimes \tau^{\prime}+\tau \otimes\left(\mathcal{L}_{v} \tau^{\prime}\right)$. This equation can be interpreted as using the representation $v \mapsto \mathcal{L}_{v}$ of vector fields as Lie derivatives after applying comultiplication $v \mapsto v \otimes 1+1 \otimes v$. Moreover, one can define inverse infinitesimal diffeomorphisms by $v \rightarrow-v$ and interpret the complex unit $1 \in K^{\otimes 0}$ as a neutral element which acts by $\mathcal{L}_{1} \equiv 1$.

These are the ingredients which can be generalized to a Hopf algebra. To this end, for an abstract Lie algebra $(\mathbb{K},[\cdot, \cdot])$, one constructs the universal enveloping algebra $U \mathbb{K}$ (also denoted as $U[\mathcal{A}]$ ) as the quotient $\mathcal{F} / \mathcal{I}$, where $\mathcal{F}$ is the free algebra generated by $(\mathbb{K}, \otimes)$ and $\mathcal{I} \subset \mathbb{F}$ the subspace containing all elements of the form $u \otimes v-v \otimes$ $u-[u, v]$. The Leibniz rule is then related to a coalgebra structure. In the example of infinitesimal diffeomorphisms, the Leibniz rule gives us the action of $\mathcal{A}$ on tensor products of functions on $\mathcal{M}$. Abstractly, we can write this action as the result of a coproduct on $U[\mathcal{A}]$, given by an algebra homomorphism $\Delta: U[\mathcal{A}] \mapsto U[\mathcal{A}] \otimes U[\mathcal{A}]$. The universal enveloping algebra of a Lie algebra $\mathcal{A}$ has a trivial coproduct given by $\Delta v=v \otimes 1+1 \otimes v$ for any $v \in \mathbb{K}$. If $U[\mathcal{A}]$ is instead equipped with a different coproduct, it is called a Hopf algebra, or quantum Lie algebra, provided that the following conditions hold: (i) Comultiplication is coassociative: $(\Delta \otimes 1) \circ \Delta=(1 \otimes \Delta) \circ \Delta$. (ii) There is an inversion map or antipode $S: U[\mathcal{A}] \rightarrow U[\mathcal{A}]$ which is an antihomomorphism. (iii) The unit (or neutral) element $\mathbb{I} \in$ $U[\mathcal{A}]$ is complemented by a co-unit $\epsilon: U[\mathcal{A}] \mapsto \mathbb{C}$ which is a homomorphism. (iv) These maps are compatible with the multiplication map $\mu: U[\mathcal{A}] \otimes U[\mathcal{A}] \rightarrow U[\mathcal{A}]$ in the sense that $\mu \circ(S \otimes 1) \circ \Delta=\mu \circ(1 \otimes S) \circ \Delta=\mathbb{I} \epsilon$. If these conditions are satisfied, the quintuple $H=(U[\mathcal{A}], \mu, \Delta, \epsilon, S)$ constitutes a Hopf algebra. For the universal enveloping algebra of a Lie algebra, for instance, we have $S(v)=-v$ and $\epsilon(v)=0$ for $v \in \mathcal{A}$, as well as $S(\mathbb{I})=\mathbb{I}$ and $\epsilon(\mathbb{I})=1$.

It is possible to construct a Hopf algebra from a Lie algebra by using Drinfeld twists [40,41]. The Hopf algebra of 4-dimensional diffeomorphisms has been studied in Refs. [87,88]. In the present work we are interested in deriving the deformation theory of the hypersurface Lie algebroid generating $(3+1)$-dimensional diffeomorphisms, as reviewed in the preceeding section for classical differential calculus. To this end, we derive the Hopfalgebra relations of 4-dimensional diffeomorphisms explicitly for the specific case of the Moyal-Weyl noncommutative spacetime (or $\theta$-canonical space).

\section{Noncommutative calculus}

Suppose that space-time coordinates (locally) obey a Heisenberg-like commutation relation, such that the commutator between coordinates is analogous to the commutation relation between configuration and momentum variables in quantum mechanics:

$$
\left[\hat{x}^{\mu}, \hat{x}^{\nu}\right]=i \theta^{\mu \nu} .
$$


We restrict our attention to the case in which $\theta^{\mu \nu}=-\theta^{\nu \mu}$ is constant and real. (It is an antisymmetric matrix of numbers and does not depend on coordinate operators.) This is the so-called Moyal-Weyl spacetime [124]. As a result of assuming such a nontrivial commutator, the multiplication between functions no longer enjoys the commutativity property:

$$
F(\hat{x}) G(\hat{x}) \neq G(\hat{x}) F(\hat{x}) .
$$

By means of a Moyal-Weyl map $\Omega$ [124], it is possible to establish a correspondence between the object $F(\hat{x}) G(\hat{x})$ and a suitably modified multiplication rule $f(x) \star g(x)$ between functions of coordinates,

$$
F(\hat{x}) G(\hat{x})=: \Omega(f(x) \star g(x)) .
$$

One can show that there are infinitely many possible choices for $\Omega$ that reproduce standard expressions in the appropriate limit. Thus, given a noncommutative algebra there is no unique Weyl map.

For the constant $\theta$ case, the most straightforward choice is

$$
f(x) \star g(x)=f(x) e^{-\frac{1}{2} i \tilde{\partial}_{\alpha} \theta^{\alpha \beta} \vec{\partial}_{\beta}} g(x) .
$$

We follow the usual quantum-group notation and introduce the twist element $\mathcal{F}=f^{\alpha} \otimes f_{\alpha}:=e^{\frac{1}{2} i^{\alpha \beta} \partial_{\alpha} \otimes \partial_{\beta}} \in U[\mathcal{A}] \otimes$ $U[\mathcal{A}]$ and its inverse, $\mathcal{F}^{-1}=\bar{f}^{\alpha} \otimes \bar{f}_{\alpha}:=e^{-\frac{1}{2} i \theta^{\alpha \beta} \partial_{\alpha} \otimes \partial_{\beta}}$. Here, $\alpha$ is used as a multi-index as shown by an expansion of the exponential function:

$$
\begin{aligned}
\mathcal{F}= & 1+\frac{1}{2} i \theta^{\alpha \beta} \partial_{\alpha} \otimes \partial_{\beta}-\frac{1}{8} \theta^{\alpha_{1} \beta_{1}} \theta^{\alpha_{2} \beta_{2}} \partial_{\alpha_{1}} \partial_{\alpha_{2}} \otimes \partial_{\beta_{1}} \partial_{\beta_{2}}+\cdots \\
& +\frac{1}{n !}(i / 2)^{n} \theta^{\alpha_{1} \beta_{1}} \cdots \theta^{\alpha_{n} \beta_{n}} \partial_{\alpha_{1}} \cdots \partial_{\alpha_{n}} \otimes \partial_{\beta_{1}} \cdots \partial_{\beta_{n}}+\cdots
\end{aligned}
$$

We can then write

$$
f_{\alpha}=\sum_{n=0}^{\infty} \frac{(i / 2)^{n / 2}}{\sqrt{n !}} \partial_{\alpha_{1}} \cdots \partial_{\alpha_{n}},
$$

raise the multi-index using $\theta^{\alpha_{1} \beta_{1}} \cdots \theta^{\alpha_{n} \beta_{n}}$, and write more compactly

$$
f(x) \star g(x)=: \bar{f}^{\alpha}(f(x)) \bar{f}_{\alpha}(g(x)) .
$$

Thus, the identity or neutral element of the tensor product of algebras, $U[\mathcal{A}] \otimes U[\mathcal{A}]$, is given by $1 \otimes 1=\mathcal{F}^{-1} \mathcal{F}=$ $\bar{f}^{\beta} f^{\alpha} \otimes \bar{f}_{\beta} f_{\alpha}$. In this notation, when we omit the right (or left) arrow over partial derivatives $\vec{\partial}_{\alpha}$ (or $\overleftarrow{\partial}_{\alpha}$ ), the derivative on the left-hand side of a tensor product acts to the left while the derivative on the right-hand side acts on functions standing to the right of the star.
The $\star$-product allows one to map the product of operator-valued functions to a modified product between functions. The product is noncommutative but still obeys associativity:

$$
(f \star g) \star h=f \star(g \star h) .
$$

In terms of the twist and the coproduct, the associative property can be expressed as

$$
\mathcal{F}_{12}(\Delta \otimes 1) \mathcal{F}=\mathcal{F}_{23}(1 \otimes \Delta) \mathcal{F},
$$

or equivalently

$$
f^{\beta} f_{1}^{\alpha} \otimes f_{\beta} f_{2}^{\alpha} \otimes f_{\alpha}=f^{\alpha} \otimes f_{\alpha}^{1} f^{\beta} \otimes f_{\beta} f_{\alpha}^{2} .
$$

In the former equation we have used $\mathcal{F}_{12}=\mathcal{F} \otimes 1=$ $f^{\alpha} \otimes f_{\alpha} \otimes 1 \in U[\mathcal{A}] \otimes U[\mathcal{A}] \otimes U[\mathcal{A}] \quad$ and $\quad \mathcal{F}_{23}=1 \otimes$ $\mathcal{F}=1 \otimes f^{\alpha} \otimes f_{\alpha} \in U[\mathcal{A}] \otimes U[\mathcal{A}] \otimes U[\mathcal{A}] . \quad$ An analogous property holds for the inverse twist element. (These identities can be confirmed by using the explicit expression for the twist $\mathcal{F}=e^{\frac{i}{2} \alpha \beta} \partial_{\alpha} \otimes \partial_{\beta}$ and its inverse $\mathcal{F}^{-1}=$ $e^{-\frac{i}{2} \theta^{\alpha \beta} \partial_{\alpha} \otimes \partial_{\beta}}$.) A second property which $\mathcal{F}$ has to satisfy is

$$
(\epsilon \otimes 1) \circ \mathcal{F}=1=(1 \otimes \epsilon) \circ \mathcal{F} .
$$

We now wish to define a commutator element in $U[\mathcal{A}] \otimes U[\mathcal{A}]$, which is called the R-matrix and allows us to make a permutation of the functions we are (star) multiplying. We define

$$
f \star g=: \bar{R}^{\alpha}(g) \star \bar{R}_{\alpha}(f),
$$

where $R^{-1}=\bar{R}^{\alpha} \otimes \bar{R}_{\alpha}$. In order to find the R-matrix in explicit form, we write

$$
\begin{aligned}
f \star g & =\bar{f}^{\alpha}(f) \bar{f}_{\alpha}(g)=\bar{f}_{\beta} f_{\gamma} \bar{f}^{\alpha}(f) \bar{f}^{\beta} f^{\gamma} \bar{f}_{\alpha}(g) \\
& =\bar{f}^{\beta}\left(f^{\gamma} \bar{f}_{\alpha}(g)\right) \bar{f}_{\beta}\left(f_{\gamma} \bar{f}^{\alpha}(f)\right) \\
& =\bar{f}^{\beta}\left(\bar{R}^{\alpha}(g)\right) \bar{f}_{\beta}\left(\bar{R}_{\alpha}(f)\right)=\bar{R}^{\alpha}(g) \star \bar{R}_{\alpha}(f),
\end{aligned}
$$

with $\bar{R}^{\alpha} \otimes \bar{R}_{\alpha}:=f^{\gamma} \bar{f}_{\alpha} \otimes f_{\gamma} \bar{f}^{\alpha}$. Here we used only the representation of the identity in the second step. As a result, the R-matrix is given by $R=R^{\alpha} \otimes R_{\alpha}=$ $f_{\gamma} \bar{f}^{\alpha} \otimes f^{\gamma} \bar{f}_{\alpha}$. In particular, for the Moyal-Weyl spacetime we are considering here, one can verify

$$
R=e^{i \theta^{\alpha \beta} \partial_{\alpha} \otimes \partial_{\beta}}, \quad R^{-1}=e^{-i \theta^{\alpha \beta} \partial_{\alpha} \otimes \partial_{\beta}} .
$$

Using twist properties, the Yang-Baxter equation $R_{12} R_{13} R_{23}=R_{23} R_{13} R_{12}$ follows. 


\section{Twisted and deformed diffeomorphisms}

Before turning to diffeomorphisms, we introduce the notion of a Lie bracket. We define two different generalizations of standard brackets between two fields: the $\star$-Lie bracket $[,]_{\star}$ and the Moyal bracket $[*$, . In the next sections, we will define the action of twisted and deformed diffeomorphisms on single fields by using these two brackets. The $\star$-Lie bracket between two generic vector fields, $v_{1}$ and $v_{2}$, is defined as

$$
\left[v_{1}, v_{2}\right]_{\star}:=v_{1} \star v_{2}-\bar{R}^{\alpha}\left(v_{2}\right) \star \bar{R}_{\alpha}\left(v_{1}\right) .
$$

In components,

$$
\left[v_{1}, v_{2}\right]_{\star}^{\mu}=v_{1}^{\rho} \star \partial_{\rho} v_{2}^{\mu}-f^{\gamma} \bar{f}_{\alpha} v_{2}^{\rho} \star \partial_{\rho} f_{\gamma} \bar{f}^{\alpha} v_{1}^{\mu} .
$$

Given this definition we can show that

$$
\left[v_{1}, v_{2}\right]_{\star}=\left[\overline{f^{\alpha}}\left(v_{1}\right), \bar{f}_{\alpha}\left(v_{2}\right)\right],
$$

where on the right-hand side we have the classical Lie bracket: We compute

$$
\begin{aligned}
{\left[v_{1}, v_{2}\right]_{\star} } & =v_{1} \star v_{2}-\bar{R}^{\alpha}\left(v_{2}\right) \star \bar{R}_{\alpha}\left(v_{1}\right) \\
& =\bar{f}^{\alpha}\left(v_{1}\right) \bar{f}_{\alpha}\left(v_{2}\right)-f^{\gamma} \bar{f}_{\alpha} \bar{f}^{\beta}\left(v_{2}\right) f_{\gamma} \bar{f}^{\alpha} \bar{f}_{\beta}\left(v_{1}\right) \\
& =\overline{f^{\alpha}}\left(v_{1}\right) \bar{f}_{\alpha}\left(v_{2}\right)-\bar{f}_{\alpha}\left(v_{2}\right) \bar{f}^{\alpha}\left(v_{1}\right) \\
& =\left[\bar{f}^{\alpha}\left(v_{1}\right), \bar{f}_{\alpha}\left(v_{2}\right)\right] .
\end{aligned}
$$

This $\star$-Lie bracket satisfies the following modification of the Jacobi identity

$\left[v_{1},\left[v_{2}, v_{3}\right]_{\star}\right]_{\star}=\left[\left[v_{1}, v_{2}\right]_{\star}, v_{3}\right]_{\star}+\left[\bar{R}^{\alpha}\left(v_{2}\right),\left[\bar{R}_{\alpha}\left(v_{1}\right), v_{3}\right]_{\star}\right]_{\star}$.

Alternatively, we can define what we call the Moyal bracket:

$$
\left[v_{1}^{\star}, v_{2}\right]:=v_{1} \star v_{2}-v_{2} \star v_{1} \text {. }
$$

It obeys the usual Jacobi identity

$$
\left[v_{1}^{\star},\left[v_{2}, v_{3}\right]\right]=\left[\left[v_{1}^{\star}, v_{2}\right]^{\star}, v_{3}\right]+\left[v_{2},\left[v_{1}^{\star}, v_{3}\right]\right],
$$

in contrast to $\star$-Lie brackets. Indeed, it is immediate to notice that $\left[v_{1}, v_{2}\right]_{\star} \neq\left[v_{1}^{\star}, v_{2}\right]$. This result will be at the root of the difference between twisted diffeomorphisms and deformed diffeomorphisms. We anticipate that the former do not change the action on single fields but have a modified Leibniz rule, while the latter retain the Leibniz rule but act on single fields in a nonstandard way. As mentioned, to have a consistent differential structure, we will then have to change the definition of deformed diffeomorphisms in such a way that there is a deformation not only of the action but also of the Leibniz rule. We also mention that the Moyal bracket allows us to map Eq. (20) into $\left[x^{\mu \star}, x^{\nu}\right]=i \theta^{\mu \nu}$. Thus, this bracket is needed to provide a representation of Eq. (20) on manifolds equipped with the nonstandard product of Eq. (23).

Another property which we will extensively use is $\partial_{\mu} \star f=\partial_{\mu} f$, which is a direct consequence of Eq. (23) with constant $\theta$, and, consequently, $\partial_{\mu}(f \star g)=$ $\left(\partial_{\mu} f\right) \star g+f \star\left(\partial_{\mu} g\right)$. Finally, as first discussed for instance in Ref. [87], the $\star$-tensor product of tensors, which is needed to have a noncommutative differential calculus together with the generalizations of Lie brackets defined above, is given by

$$
\tau \otimes_{\star} \tau^{\prime}=\bar{f}^{\alpha}(\tau) \otimes \bar{f}_{\alpha}\left(\tau^{\prime}\right) .
$$

The tensor product is therefore twisted just as the pointwise product of functions.

Let us now discuss the two different paths to treating diffeomorphisms on $\mathcal{A}$, that is twisted and deformed (or $\star-$ ) diffeomorphisms. As already stressed, for the latter, which we here study for the first time, we will consider two different candidates: either with trivial or nontrivial coproduct. The general idea consists in finding a meaningful generalization of general covariance to noncommutative manifolds, where noncommutative manifolds are quantizations of classical smooth manifolds in the sense that the product of fields evaluated at a spacetime point is noncommutative and is given by the $\star$-product.

\section{TWISTED DIFFEOMORPHISMS}

We return to the derivation of hypersurface-deformation brackets, but now in a generalization to noncommutative calculus.

\section{A. Lie derivative}

We start by analyzing twisted diffeomorphisms, which have already been introduced, for instance in Ref. [87], in their 4-dimensional form. Here, we will focus on their $3+1$-dimensional version. We shall see that most of the statements made in Ref. [87] apply also for the twisting of hypersurface-deformation brackets.

Consider a generic tensor $u$. On a commutative space, it transforms as $u^{\prime}=u+\delta_{v} u=u+\mathcal{L}_{v} u$ under infinitesimal diffeomorphisms generated by the vector field $v=v^{\mu} \partial_{\mu}$. As usual, $\mathcal{L}_{v} u$ is the Lie derivative of $u$ along $v$. It is possible to represent standard diffeomorphisms on $\mathcal{A}$ by means of twisting. For a function $u$, we write

$$
\begin{aligned}
\delta_{v} u & =\mathcal{L}_{v} u=v^{\rho} \partial_{\rho} u=f^{\beta} \bar{f}^{\alpha}\left(v^{\rho} \partial_{\rho}\right) f_{\beta} \bar{f}_{\alpha}(u) \\
& =\left(f^{\beta}\left(v^{\rho} \partial_{\rho}\right) f_{\beta}\right) \star u=\mathcal{L}_{v^{\star}} \triangleright u
\end{aligned}
$$

We have inserted the representation of the identity in terms of the twist and its inverse, and defined 


$$
\begin{aligned}
v^{\star}:= & f^{\beta}(v) f_{\beta}=\sum_{n}\left(-\frac{i}{2}\right)^{n} \frac{1}{n !} \theta^{\mu_{1} \nu_{1}} \ldots \theta^{\mu_{n} \nu_{n}} \\
& \times\left(\partial_{\mu_{1}} \ldots \partial_{\mu_{n}} v^{\rho}\right) \partial_{\nu_{1}} \ldots \partial_{\nu_{n}} \partial_{\rho}
\end{aligned}
$$

as an element of $U[\mathcal{A}]$. The application of $\mathcal{L}_{v^{\star}}$ is what we call an infinitesimal twisted diffeomorphism.

For a vector field $u^{\mu}$, we proceed in a similar way and write

$$
\begin{aligned}
\mathcal{L}_{v} u^{\mu} & =v^{\rho} \partial_{\rho} u^{\mu}-\left(\partial_{\rho} v^{\mu}\right) u^{\rho} \\
& =f^{\beta}\left(v^{\rho} \partial_{\rho}\right) f_{\beta} \star u^{\mu}-\partial_{\rho}\left(f^{\beta}\left(v^{\mu}\right) f_{\beta}\right) \star u^{\rho} \\
& =\left(v^{\rho} \partial_{\rho}\right)^{\star} \star u^{\mu}-\left(\partial_{\rho} v^{\star}\right)^{\mu} \star u^{\rho},
\end{aligned}
$$

always keeping $v$ to the left of $u$. In the second term, we may change the ordering by applying the $R$-matrix,

$$
\begin{aligned}
\mathcal{L}_{v} u^{\mu} & =v^{\star} \star u^{\mu}-\bar{R}^{\alpha}\left(u^{\rho}\right) \star \partial_{\rho} \bar{R}_{\alpha}\left(v^{\star}\right)^{\mu} \\
& =\left[v^{\star}, u\right]_{\star},
\end{aligned}
$$

in order to derive a relationship with Eq. (33). However, this notation has to be treated with some care because $\left(v^{\star}\right)^{\mu}$ is not a function but acts to the left on $u^{\rho}$ in the second term of the commutator.

The same procedure can be used to derive the Lie derivative of an arbitrary tensor (density), rewriting the classical relationships in such a way that components of $v$ (the vector field along which we take the Lie derivative) always stay on the left. For instance, for the metric tensor $g_{\mu \nu}$, we have

$$
\mathcal{L}_{v} g_{\mu \nu}=v^{\star} \star g_{\mu \nu}+\left(\partial_{\mu} v^{\star \rho}\right) \star g_{\rho \nu}+\left(\partial_{\nu} v^{\star \rho}\right) \star g_{\mu \rho} .
$$

\section{B. Twisted Gaussian system}

With these preparations, we can introduce the notion of a noncommutative Gaussian system for twisted diffeomorphisms. From the point of view of hypersurface deformations, the main property of a Gaussian system should be that it leads to constant components $g_{0 \mu}$ of the metric. In this way, the lapse function and shift vector in the background metric are fixed, and it becomes possible to isolate the role of lapse and shift as generators of hypersurface deformations. The simplest choice of constant background lapse and shift that is compatible with a non-degenerate metric of Lorentzian signature is $g_{00}=-1$ and $g_{0 i}=0$ for $i \neq 0$.

We need to show that there is a choice of coordinates on a noncommutative manifold such that the metric is Gaussian in the specified sense. We do so by assuming the classical Gaussian system under the standard product of functions or coordinates, and showing that there is a frame in which the required properties are satisfied also for a noncommutative product and twisted diffeomorphisms. In particular, the classical system provides us with a time coordinate $t$ such that $n=\mathrm{d} t$ is the co-normal to spatial hypersurfaces $t=$ constant. The same 1 -form is a conormal on a noncommutative manifold with twisted diffeomorphisms: For a vector field $X$ tangential to a spatial hypersurface and $n=\mathrm{d} t$, we have

$$
X^{\mu} \star n_{\mu}=i_{X^{\star}} \star \mathrm{d} n=\mathcal{L}_{X^{\star}} \triangleright t=X^{\mu} \partial_{\mu} t=0 .
$$

The Lie derivative along $X^{\star}$ is equal to the classical Lie derivative because all higher-derivative terms in (42) vanish when acting on a linear function such as $t$. In a Gaussian frame, the co-normal therefore has constant components, and so does the normal $n^{\mu}=g^{\mu \nu} \star n_{\mu}=g^{\mu \nu} n_{\mu}$ because higher derivatives in the star product vanish when applied to a constant $n_{\mu}$. Here we introduced the inverse metric $g^{\nu \alpha} \star g_{\alpha \mu}=\delta_{\mu}^{\alpha}$, defining the inverse metric by its action from the left (alternatively one can define the inverse bythe-right) [87].

The normal is therefore normalized with respect to the noncommutative system, in the following sense:

$$
\begin{aligned}
i_{n^{\star}} \star g \star i_{n} & =n^{\star \mu} \star g_{\mu \nu} \star n^{\nu}=f^{\alpha} n^{\mu} f_{\alpha} \star g_{\mu \nu} \star n^{\nu} \\
& =n^{\mu} g_{\mu \nu} \star n^{\nu}=n_{\nu} \star n^{\nu}=n_{\nu} n^{\nu}=-1 .
\end{aligned}
$$

In a classical Gaussian system, we have $n^{\mu} \nabla_{\mu} n^{\nu}=0$ because worldlines normal to spatial hypersurfaces are geodesics. In a Gaussian frame, all contributions from connection components in this equation are zero because the only relevant ones,

$$
\Gamma_{0 \mu}^{0}=\frac{1}{2} g^{0 \alpha}\left(\partial_{\mu} g_{0 \alpha}+\partial_{0} g_{\mu \alpha}-\partial_{\alpha} g_{0 \mu}\right)=0,
$$

vanish identically for a Gaussian metric. The equation $n^{\mu} \nabla_{\mu} n^{\nu}=0$ is therefore equivalent to $n^{\mu} \partial_{\mu} n^{\nu}=0$ in a Gaussian system. The same equation is true in the form $n^{\mu} \star \partial_{\mu} n^{\nu}=0$ for a noncommutative Gaussian system because, as we just showed, the components of $n^{\mu}$ are still constant. From this equation, we can derive $n^{\mu} \star \nabla_{\mu} \star$ $n^{\nu}=0$ using the definition of the noncommutative Christoffel connection from [87], which gives

$$
\begin{aligned}
\Gamma_{0 \mu}^{0} & =\frac{1}{2} g^{0 \alpha} \star\left(\partial_{\mu} g_{0 \alpha}+\partial_{0} g_{\mu \alpha}-\partial_{\alpha} g_{0 \mu}\right) \\
& =\frac{1}{2} g^{0 \alpha} \star \partial_{0} g_{\mu \alpha}=0,
\end{aligned}
$$

for the relevant connection components.

It will be convenient to do calculations of the hypersurface-deformation brackets in a Gaussian frame. However, whenever possible, we will not make explicit use of the fact that normal components are constant in 
order to display all relevant star products. In particular, in order to be as general as possible, we will derive differential equations for the normal and tangential components of a Gaussian vector field without using constant components of the normal. We will see that a counterterm is then required in the classical Gaussian condition. We then analyze these differential equations using all the properties of a Gaussian frame, including the constant nature of components of the normal. This step will allow us to show that there is a well-posed initial-value problem and a set of algebroid brackets which depend only on hypersurface data.

\section{Gaussian condition}

We are interested in deriving properties of hypersurface deformations in noncommutative space-time, with possible modifications of the action of twisted diffeomorphisms. We modify the classical expression used to define a Gaussian vector field as follows: Instead of $i_{n} \mathcal{L}_{v} g=0$, we require that

$$
\left(\mathcal{L}_{v^{\star}} \triangleright g\right) \star i_{n}=0
$$

We act with $i_{n}$ from the right in order to make sure that it stands next to the metric, without components of $v^{\star}$ in between. Classically, we say that $v$ is Gaussian if a diffeomorphism of the metric along the direction given by $v$ does not have a normal component. We have generalized this statement by saying that the twisted infinitesimal diffeomorphism of $g$, generated by $v$, gives zero if we $\star$-contract the result with the normal $n$. Since the normal components are constant, (53) is equivalent to the classical condition on Gaussian vector fields, and it is therefore consistent with the metric form of a Gaussian system.

We have that $i_{n} \mathcal{L}_{v} g=n^{\mu}\left(\mathcal{L}_{v} g\right)_{\mu \nu}$, and analogously we can write the twisted version in components as $\left(\mathcal{L}_{v^{\star}} \triangleright g\right)_{\mu \nu} \star n^{\mu}$, where the Lie derivative of the metric is given in (46) in terms of twisted diffeomorphisms. We rewrite star products using (25), for instance $\left(v^{\rho}\right)^{\star} \star \partial_{\rho} g=$ $\bar{f}^{\alpha}\left(\left(v^{\rho}\right)^{\star} \partial_{\rho}\right) \bar{f}_{\alpha}(g)$ in the first term, and therefore obtain the Gaussian condition for $v$ as

$$
\left(\mathcal{L}_{\bar{f}^{\alpha}\left(v^{\star}\right) \bar{f}_{\alpha}} g\right) \star i_{n}=0 .
$$

The next step is to try and obtain relations for the normal and tangential components of the $\star$-Lie bracket between the normal $n$ and the Gaussian vector field $v$. In doing that, we will try to follow as close as possible the steps of the derivation for the commutative case.

First, we would like to compute $\mathcal{L}_{\bar{f}^{\alpha}\left(v^{\star}\right) \bar{f}_{\alpha}}\left(g \star i_{n}\right)$, or the action of the twisted Lie derivative on the $\star$-product of two fields:

$$
\begin{aligned}
\bar{f}^{\alpha}\left(v^{\star}\right) \bar{f}_{\alpha}\left(g \star i_{n}\right)= & \bar{f}^{\alpha}\left(v^{\star}\right) \bar{f}_{\alpha}\left(\bar{f}^{\beta}(g) \bar{f}_{\beta}\left(i_{n}\right)\right) \\
= & \bar{f}^{\alpha}\left(v^{\star}\right) \bar{f}_{\alpha}^{1} \bar{f}^{\beta}(g) \bar{f}_{\alpha}^{2} \bar{f}_{\beta}\left(i_{n}\right) \\
= & \bar{f}^{\alpha}\left(v^{\mu}\right)^{\star} \bar{f}_{\alpha}^{1} \bar{f}^{\beta}\left(\partial_{\mu} g_{\sigma \nu}\right) \bar{f}_{\alpha}^{2} \bar{f}_{\beta}\left(n^{\sigma}\right) \\
& +\bar{f}^{\alpha}\left(\partial_{\nu} v^{\mu}\right)^{\star} \bar{f}_{\alpha}^{1} \bar{f}^{\beta}\left(g_{\sigma \mu}\right) \bar{f}_{\alpha}^{2} \bar{f}_{\beta}\left(n^{\sigma}\right) \\
& +\bar{f}^{\alpha}\left(v^{\mu}\right)^{\star} \bar{f}_{\alpha}^{1} \bar{f}^{\beta}(g) \bar{f}_{\alpha}^{2} \bar{f}_{\beta}\left(i_{\partial_{\mu} n}\right) .
\end{aligned}
$$

Adding and subtracting the term $\bar{f}^{\alpha}\left(\partial_{\sigma} v^{\mu}\right)^{\star} \bar{f}_{\alpha}^{1} \bar{f}^{\beta}\left(g_{\nu \mu}\right) \times$ $\bar{f}_{\alpha}^{2} \bar{f}_{\beta}\left(n^{\sigma}\right)$, we obtain

$$
\begin{aligned}
& \bar{f}^{\alpha}\left(v^{\mu}\right)^{\star} \bar{f}_{\alpha}^{1} \bar{f}^{\beta}\left(\partial_{\mu} g_{\sigma \nu}\right) \bar{f}_{\alpha}^{2} \bar{f}_{\beta}\left(n^{\sigma}\right) \\
& \quad+\bar{f}^{\alpha}\left(\partial_{\nu} v^{\mu}\right)^{\star} \bar{f}_{\alpha}^{1} \bar{f}^{\beta}\left(g_{\sigma \mu}\right) \bar{f}_{\alpha}^{2} \bar{f}_{\beta}\left(n^{\sigma}\right) \\
& \quad+\bar{f}^{\alpha}\left(\partial_{\sigma} v^{\mu}\right)^{\star} \bar{f}_{\alpha}^{1} \bar{f}^{\beta}\left(g_{\nu \mu}\right) \bar{f}_{\alpha}^{2} \bar{f}_{\beta}\left(n^{\sigma}\right) \\
& \quad-\bar{f}^{\alpha}\left(\partial_{\sigma} v^{\mu}\right)^{\star} \bar{f}_{\alpha}^{1} \bar{f}^{\beta}\left(g_{\nu \mu}\right) \bar{f}_{\alpha}^{2} \bar{f}_{\beta}\left(n^{\sigma}\right) \\
& \quad+\bar{f}^{\alpha}\left(v^{\mu}\right)^{\star} \bar{f}_{\alpha}^{1} \bar{f}^{\beta}(g) \bar{f}_{\alpha}^{2} \bar{f}_{\beta}\left(i_{\partial_{\mu} n}\right) .
\end{aligned}
$$

Using both (28) and (30), for the first three terms we have

$$
\begin{aligned}
\bar{f}^{\alpha}\left(v^{\mu}\right)^{\star} & \bar{f}_{\alpha}^{1} \bar{f}^{\beta}\left(\partial_{\mu} g_{\sigma \nu}\right) \bar{f}_{\alpha}^{2} \bar{f}_{\beta}\left(n^{\sigma}\right) \\
& +\bar{f}^{\alpha}\left(\partial_{\nu} v^{\mu}\right)^{\star} \bar{f}_{\alpha}^{1} \bar{f}^{\beta}\left(g_{\sigma \mu}\right) \bar{f}_{\alpha}^{2} \bar{f}_{\beta}\left(n^{\sigma}\right) \\
& +\bar{f}^{\alpha}\left(\partial_{\sigma} v^{\mu}\right)^{\star} \bar{f}_{\alpha}^{1} \bar{f}^{\beta}\left(g_{\nu \mu}\right) \bar{f}_{\alpha}^{2} \bar{f}_{\beta}\left(n^{\sigma}\right) \\
= & \bar{f}_{1}^{\alpha} \bar{f}^{\beta}\left(v^{\mu}\right)^{\star} \bar{f}_{2}^{\alpha} \bar{f}_{\beta}\left(\partial_{\mu} g\right) \bar{f}_{\alpha}\left(i_{n}\right) \\
& +\bar{f}_{1}^{\alpha} \bar{f}^{\beta}\left(\partial_{\nu} v^{\mu}\right)^{\star} \bar{f}_{2}^{\alpha} \bar{f}_{\beta}\left(g_{\sigma \mu}\right) \bar{f}_{\alpha}\left(n^{\sigma}\right) \\
& +\bar{f}^{\beta} \bar{f}_{1}^{\alpha}\left(\partial_{\sigma} v^{\mu}\right)^{\star} \bar{f}_{2}^{\alpha} \bar{f}_{\beta}\left(g_{\nu \mu}\right) \bar{f}_{\alpha}\left(n^{\sigma}\right) \\
= & \left(\mathcal{L}_{\left.\bar{f}^{\alpha}\left(v^{\star}\right) \bar{f}_{\alpha} g\right) \star i_{n} .}\right.
\end{aligned}
$$

We write the last two terms of (56) as

$$
\begin{aligned}
& \bar{f}^{\alpha}\left(v^{\mu}\right)^{\star} \bar{f}_{\alpha}^{1} \bar{f}^{\beta}(g) \bar{f}_{\alpha}^{2} \bar{f}_{\beta}\left(i_{\partial_{\mu} n}\right) \\
& -\bar{f}^{\alpha}\left(\partial_{\sigma} v^{\mu}\right)^{\star} \bar{f}_{\alpha}^{1} \bar{f}^{\beta}\left(g_{\nu \mu}\right) \bar{f}_{\alpha}^{2} \bar{f}_{\beta}\left(n^{\sigma}\right) \\
& \left.=\bar{f}^{\alpha}\left(\bar{R}^{\gamma}(g)\right) \bar{f}_{\alpha}^{1} \bar{f}^{\beta} \bar{R}_{\gamma}\left(v^{\mu}\right)^{\star}\right) \bar{f}_{\beta} \bar{f}_{\alpha}^{2}\left(i_{\partial_{\mu} n}\right) \\
& -\bar{f}^{\alpha}\left(\bar{R}^{\gamma}\left(g_{\nu \mu}\right)\right) \bar{f}_{\alpha}^{1} \bar{f}^{\beta} \bar{R}_{\gamma}\left(\partial_{\sigma} v^{\mu}\right)^{\star} \bar{f}_{\alpha}^{2} \bar{f}_{\beta}\left(n^{\sigma}\right) \\
& =\bar{f}^{\alpha}\left(\bar{R}^{\gamma}(g)\right) \bar{f}_{\alpha}\left(\bar{R}_{\gamma}\left(\left(v^{\mu}\right)^{\star} \partial_{\mu}\right) \star i_{n}\right) \\
& -\bar{f}^{\alpha}\left(\bar{R}^{\gamma}\left(g_{\nu \mu}\right)\right) \bar{f}_{\alpha}\left(\bar{R}_{\gamma}\left(\partial_{\sigma} v^{\mu}\right)^{\star} \star\left(n^{\sigma}\right)\right)
\end{aligned}
$$

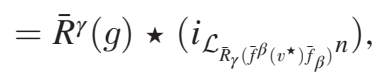

and arrive at

$$
\mathcal{L}_{\bar{f}^{\alpha}\left(v^{\star}\right) \bar{f}_{\alpha}}\left(g \star i_{n}\right)=\left(\mathcal{L}_{\bar{f}^{\alpha}\left(v^{\star}\right) \bar{f}_{\alpha}} g\right) \star i_{n}+\bar{R}^{\alpha}(g) \star\left(i_{\left.\mathcal{L}_{\bar{R}_{\alpha}\left(\bar{f} \beta\left(v^{\star}\right) \bar{f}_{\beta}\right)}\right)}\right) .
$$

We see that, as a direct consequence of loss of commutativity of the $\star$-product, the Leibniz rule does not apply. It is modified through the action of the R-matrix, as one could have anticipated. Using the above expressions we can rewrite Eq. (54) as 


$$
\begin{aligned}
\left(\mathcal{L}_{\bar{f}^{\alpha}\left(v^{\star}\right) \bar{f}_{\alpha}} g\right) \star i_{n}= & \mathcal{L}_{\bar{f}^{\alpha}\left(v^{\star}\right) \bar{f}_{\alpha}}\left(g \star i_{n}\right) \\
& -\bar{R}^{\alpha}(g) \star\left(i_{\mathcal{L}_{\bar{R}_{\alpha}\left(\bar{f}^{\beta}\left(v^{\star}\right) \bar{f}_{\beta}\right)} n}\right)=0 .
\end{aligned}
$$

The next step is an application of the Cartan identity. The validity of such an identity is usually required as an axiom, or assumed (see for instance [125]), but it is possible to prove it in the following manner. Let us make indices explicit in

$$
\begin{aligned}
\mathcal{L}_{\bar{f}^{\alpha}\left(v^{\star}\right) \bar{f}_{\alpha}}\left(g \star i_{n}\right) \\
=\bar{f}^{\alpha}\left(v^{\rho} \partial_{\rho}\right)^{\star} \bar{f}_{\alpha}\left(g_{\mu \nu} \star n^{\mu}\right) \\
=\left(v^{\rho}\right)^{\star} \star \partial_{\rho}\left(g_{\mu \nu} \star n^{\mu}\right)+\partial_{\nu}\left(v^{\rho}\right)^{\star} \star\left(g_{\rho \mu} \star n^{\mu}\right) \\
=\left(v^{\rho}\right)^{\star} \star \partial_{\rho}\left(g_{\mu \nu} \star n^{\mu}\right)+\partial_{\nu}\left(v^{\rho}\right)^{\star} \star\left(g_{\rho \mu} \star n^{\mu}\right) \\
\\
\quad+\left(v^{\rho}\right)^{\star} \star \partial_{\nu}\left(g_{\rho \mu} \star n^{\mu}\right)-\left(v^{\rho}\right)^{\star} \star \partial_{\nu}\left(g_{\rho \mu} \star n^{\mu}\right) \\
=\partial_{\nu}\left(\left(v^{\rho}\right)^{\star} \star g_{\rho \mu} \star n^{\mu}\right)+\left(v^{\rho}\right)^{\star} \star(\mathrm{d} n)_{\rho \nu},
\end{aligned}
$$

where we defined the two-form $(\mathrm{d} n)_{\rho \nu}:=\partial_{\rho}\left(g_{\mu \nu} \star n^{\mu}\right)-$ $\partial_{\nu}\left(g_{\mu \rho} \star n^{\mu}\right)$. Thus, we derived

$\mathcal{L}_{v}^{\star} \triangleright\left(g \star i_{n}\right)=i_{v^{\star}} \star \mathrm{d}\left(g \star i_{n}\right)+\mathrm{d}\left(i_{v^{\star}} \star g \star i_{n}\right)$,

commonly known as the Cartan identity.

With this result, we have

$$
\begin{aligned}
& \mathcal{L}_{\bar{f}^{\alpha}\left(v^{\star}\right) \bar{f}_{\alpha}}\left(g \star i_{n}\right)-\bar{R}^{\alpha}(g) \star\left(i_{\mathcal{L}_{\bar{R}_{\alpha}\left(\bar{f} \beta\left(v^{\star}\right) \bar{f}_{\beta}\right)} n}\right) \\
& =i_{v^{\star}} \star \mathrm{d}\left(g \star i_{n}\right)+\mathrm{d}\left(i_{v^{\star}} \star g \star i_{n}\right) \\
& -\bar{R}^{\alpha}(g) \star\left(i_{\mathcal{L}_{\bar{R}_{\alpha}\left(\bar{f} \beta\left(v^{\star}\right) \bar{f}_{\beta}\right)} n}\right)=0 .
\end{aligned}
$$

Now we use $\mathrm{d} n=\mathrm{d}\left(g \star i_{n}\right)=0$ and obtain

$$
\bar{R}^{\alpha}(g) \star\left(i_{\left.\mathcal{L}_{\bar{R}_{\alpha}\left(\overline{f^{\beta}}\left(v^{\star}\right) \bar{F}_{\beta}\right)}\right)}\right)=\mathrm{d}\left(i_{v^{\star}} \star g \star i_{n}\right) .
$$

\section{Decomposition}

Decomposing $v^{\star}$ into components normal and tangential to hypersurfaces, $v^{\star}=\left(N^{\star} \star n\right)^{\star}+\left(M^{\star} \star X\right)^{\star}$ (with $N^{\star}:=f^{\alpha}(N) f_{\alpha}$ and $\left.M^{\star}:=f^{\alpha}(M) f_{\alpha}\right)$, we write

$$
\begin{aligned}
& \bar{R}^{\alpha}(g) \star\left(i_{\mathcal{L}_{\bar{R}_{\alpha}\left(\bar{f} \beta\left(N^{\star} \star n\right)^{\star} \bar{f}_{\beta}\right)} n}\right) \\
& \quad+\bar{R}^{\alpha}(g) \star\left(i_{\mathcal{L}_{\bar{R}_{\alpha}\left(\overline{f^{\beta}}\left(M^{\star} \star X\right)^{\star} \bar{F}_{\beta}\right)} n}\right)=-\mathrm{d} N^{\star},
\end{aligned}
$$

where we have used the relations

$$
i_{n^{\star}} \star g \star i_{n}=-1 \quad i_{X^{\star}} \star g \star i_{n}=0 ;
$$

see (48).
Writing indices explicitly,

$$
\begin{aligned}
& \bar{R}^{\alpha}\left(g_{\nu \mu}\right) \star\left[\bar{R}_{\alpha} \bar{f}^{\beta}\left(N^{\star} \star n^{\rho}\right)^{\star} \bar{f}_{\beta}\left(\partial_{\rho} n^{\mu}\right)\right. \\
& \quad-\bar{R}_{\alpha} \bar{f}^{\beta} \partial_{\rho}\left(N^{\star} \star n^{\mu}\right)^{\star} \bar{f}_{\beta}\left(n^{\rho}\right) \\
& \quad+\bar{R}_{\alpha} \bar{f}^{\beta}\left(M^{\star} \star X^{\rho}\right)^{\star} \bar{f}_{\beta}\left(\partial_{\rho} n^{\mu}\right) \\
& \left.\quad-\bar{R}_{\alpha} \bar{f}^{\beta} \partial_{\rho}\left(M^{\star} \star X^{\mu}\right)^{\star} \bar{f}_{\beta}\left(n^{\rho}\right)\right]=-\partial_{\nu} N^{\star},
\end{aligned}
$$

where we left implicit only the internal index $a$ in $M \star X^{\mu} \equiv M^{a} \star X_{a}^{\mu}$. Using Eq. (30), we have

$$
\begin{aligned}
& \bar{R}^{\alpha}\left(g_{\nu \mu}\right) \star\left[\bar{R}_{\alpha} \bar{f}^{\beta}\left(N^{\star} \star n^{\rho} \partial_{\rho}\right)^{\star} \bar{f}_{\beta}\left(n^{\mu}\right)\right. \\
& \quad-\bar{f}^{\beta}\left(\bar{R}^{\gamma}\left(n^{\rho} \partial_{\rho}\right)\right) \bar{f}_{\beta}\left(\bar{R}_{\alpha} \bar{R}_{\gamma}\left(N^{\star} \star n^{\mu}\right)^{\star}\right) \\
& \quad+\bar{R}_{\alpha} \bar{f}^{\beta}\left(M^{\star} \star X^{\rho} \partial_{\rho}\right)^{\star} \bar{f}_{\beta}\left(n^{\mu}\right) \\
& \left.\quad-\bar{f}^{\beta}\left(\bar{R}_{\alpha} \bar{R}^{\gamma}\left(n^{\rho} \partial_{\rho}\right)\right) \bar{f}_{\beta}\left(\bar{R}_{\gamma}\left(M^{\star} \star X^{\mu}\right)^{\star}\right)\right]=-\partial_{\nu} N^{\star},
\end{aligned}
$$

and finally, recalling Eq. (33),

$$
\begin{aligned}
& \bar{R}^{\alpha}\left(g_{\nu \mu}\right) \star\left(\left[\bar{R}_{\alpha}\left(N^{\star} \star n\right)^{\star}, n\right]_{\star}^{\mu}\right. \\
& \left.\quad+\left[\bar{R}_{\alpha}\left(M^{\star} \star X\right)^{\star}, n\right]_{\star}^{\mu}\right)=-\partial_{\nu} N^{\star} .
\end{aligned}
$$

So far, following Refs. [87,88], we have defined twisted (four) diffeomorphisms by a representation of the infinitesimal diffeomorphisms of classical differential manifolds on noncommutative manifolds or, rather, on manifolds equipped with a non-trivial $\star$-multiplication rule (23). As a consequence, they have an undeformed action on single fields or tensors but, due to the Moyal $\star$-product, act nontrivially on products of two or more objects. Thus, twisting diffeomorphisms corresponds to mapping them to the Moyal space (or, more generally, to a manifold with noncommutative products). In order to find formulas relating the lapse function and shift vector components, it will be more useful to rewrite the relation (68) as one on the commutative classical manifold in an intermediate step. We will then represent the final hypersurface-deformation brackets on the Moyal space in order to obtain a twisted version of the HDA.

Using the definition of the R-matrix as well as that of the $\star$-Lie bracket, we rewrite Eq. (68) as

$$
\begin{aligned}
-\partial_{\nu} N^{\star}= & \left(N n^{\rho} g_{\nu \mu}\right) \star \partial_{\rho} n^{\mu}-\left(\partial_{\rho}\left(N n^{\mu}\right) g_{\nu \mu}\right) \star n^{\rho} \\
& +\left(M^{\rho} g_{\nu \mu}\right) \star \partial_{\rho} n^{\mu}-\left(\partial_{\rho} M^{\mu} g_{\nu \mu}\right) \star n^{\rho} \\
= & g_{\mu \nu}^{\star} \star N^{\star} \star\left(n^{\rho} \star \partial_{\rho} n^{\mu}-\left(\partial_{\rho} n^{\mu}\right) \star n^{\rho}\right) \\
& -g_{\mu \nu}^{\star} \star n^{\mu} \star \partial_{\rho} N^{\star} \star n^{\rho} \\
& +g_{\nu \mu}^{\star} \star M^{\rho} \star \partial_{\rho} n^{\mu}-g_{\nu \mu}^{\star} \star \partial_{\rho} M^{\mu} \star n^{\rho} .
\end{aligned}
$$

We can now use the constant nature of $n_{\mu}$ in a Gaussian frame, so that $n^{\rho}$ star-commutes with any function and the partial gradient $\partial_{\rho} n^{\mu}=0$ vanishes. Multiplying both sides of (69) by $n^{\nu}$, we have 
$-n^{\nu} \star \partial_{\nu} N^{\star}-\partial_{\nu} N^{\star} \star n^{\nu}=-n^{\nu} \star g_{\nu \mu}^{\star} \star \partial_{\rho} M^{\mu} \star n^{\rho}$,

where we also used $n^{\mu} \star n_{\mu}=-1$. Applying the product rule in

$$
\begin{aligned}
0= & n^{\rho} \star \partial_{\rho}\left(n_{\mu} \star M^{\mu}\right)=\left(n^{\rho} \star \partial_{\rho} n_{\mu}\right) \star M^{\mu}+n_{\mu} \star\left(n^{\rho} \star \partial_{\rho} M^{\mu}\right) \\
& +\left(n_{\mu} \star n^{\rho}-n^{\rho} \star n_{\mu}\right) \star \partial_{\rho} M^{\mu},
\end{aligned}
$$

and using $n^{\nu} \star X_{\nu}=0$ as well as the vanishing star commutator $n_{\mu} \star n^{\rho}-n^{\rho} \star n_{\mu}=0$ of the constant $n_{\mu}$, implies that $n^{\rho} \star \partial_{\rho} M^{\mu}=0$. Thus, we finally obtain

$0=-n^{\nu} \star \partial_{\nu} N^{\star}-\partial_{\nu} N^{\star} \star n^{\nu}=-2 n^{\nu} \partial_{\nu} N^{\star}=-2 n^{\nu} \partial_{\nu} N$.

In the last step, we have mapped the expression back to the commutative space and, therefore, multiplication is the usual commutative rule.

The tangential projection of Eq. (68) is made in a similar way. By $\star$-multiplying with $q^{a b}$, we have

$$
[n, M]_{\star}^{a}=q^{a b} \star \partial_{b} N^{\star} .
$$

Lapse $N$ and shift $M^{a}$ are subject to the same type of partial differential equations as in the classical derivation. Therefore, they are extendable to a space-time neighborhood of a spatial hypersurface and can be used in the Lie brackets of Gaussian space-time vector fields.

\section{E. Brackets}

We are now ready to evaluate the $\star$-Lie bracket of spacetime vector fields. We calculate the $\star$-product between the $\star$-Lie bracket $\left[v_{1}^{\star}, v_{2}^{\star}\right]_{\star}^{\mu}$ and an arbitrary scalar function $f$ for twisted diffeomorphisms,

$$
\begin{aligned}
{\left[v_{1}^{\star}, v_{2}^{\star}\right]_{\star}^{\mu} \star f=} & \left(\left(v_{1}^{\rho}\right)^{\star} \star \partial_{\rho}\left(v_{2}^{\mu}\right)^{\star}-\bar{R}^{\alpha}\left(v_{2}^{\rho}\right)^{\star} \star \bar{R}_{\alpha}\left(\partial_{\rho} v_{1}^{\mu}\right)^{\star}\right) \star \partial_{\mu} f \\
= & v_{1}^{\rho} \partial_{\rho} v_{2}^{\mu} \partial_{\mu} f-\partial_{\rho} v_{1}^{\mu} v_{2}^{\rho} \partial_{\mu} f \\
= & \left(N_{1} n^{\rho}+M_{1}^{\rho}\right) \partial_{\rho}\left(N_{2} n^{\mu}+M_{2}^{\mu}\right) \partial_{\mu} f \\
& -\partial_{\rho}\left(N_{1} n^{\mu}+M_{1}^{\mu}\right)\left(N_{2} n^{\rho}+M_{2}^{\rho}\right) \partial_{\mu} f \\
= & \left(N_{1} n^{\rho} \partial_{\rho} N_{2}-\partial_{\rho} N_{1} N_{2} n^{\rho}\right) n^{\mu} \partial_{\mu} f \\
& +\left(\mathcal{L}_{M_{1}} N_{2}-\mathcal{L}_{M_{2}} N_{1}\right) n^{\mu} \partial_{\mu} f \\
& +\left[M_{1}, M_{2}\right]^{\mu} \partial_{\mu} f+N_{1}\left[n, M_{2}\right]^{\mu} \partial_{\mu} f \\
& -N_{2}\left[n, M_{1}\right]^{\mu} \partial_{\mu} f
\end{aligned}
$$

and extract normal and tangential terms and using the above relations for $[n, M]$ :

$$
\begin{gathered}
{\left[\left(0, M_{1}^{a}\right),\left(0, M_{2}^{b}\right)\right]=\left(0, \mathcal{L}_{M_{1}} M_{2}\right),} \\
{\left[(N, 0),\left(0, M^{a}\right)\right]=\left(-\mathcal{L}_{M} N, 0\right),} \\
{\left[\left(N_{1}, 0\right),\left(N_{2}, 0\right)\right]=\left(0,\left(N_{1} \partial_{b} N_{2}-N_{2} \partial_{b} N_{1}\right) q^{a b}\right) .}
\end{gathered}
$$

The fact that this result coincides with Eq. (17) confirms the claim [87] that twisted noncommutative gravity with the Moyal product has the same symmetry algebra as classical GR. Thus, the only deformations of symmetries are encoded in the coalgebraic sector where, due to the nonstandard multiplication, the Leibniz rule does not apply. Having a closed and consistent set of brackets also ensures that noncommutative gravity possesses the same number of d.o.f. as GR, as one should expect. We shall see that this statement remains true also for deformed diffeomorphism symmetries, in which case the HDA does receive $\star$-product deformations.

Once one has obtained the Poisson brackets for general coordinate transformations, it is of interest to study their Minkowski (or flat) limit. In this way, one restricts the set of diffeomorphisms and only allows a subset of coordinate transformations, which are the isometries of Minkowski spacetime. In terms of hypersurface deformations, this restriction can be implemented by using the Euclidean spatial metric and requiring lapse and shift to be linear in space coordinates, of the form $N=\alpha+\alpha_{i} x^{i}$ and $M^{a}=$ $\beta^{a}+R_{b}^{a} x^{b}$ ( $R_{a b}$ being a matrix of rotations in space). The interested reader can take a look at Refs. $[93,115,119]$ for the Minkowski limit of the HDA and its deformations. Here, as expected, we find that the twisted HDA has no deformations compared with the standard version of GR. It is then not difficult to show that, after the specified restrictions, the resulting Poincaré algebra is also unmodified. On the other hand, one can expect that the action of Poincare generators on products of functions will be nontrivial as a result of the presence of a noncommutative multiplication rule. This is consistent with the known fact that the symmetry algebra dual to the Moyal-Weyl space-time is the so-called $\theta$ Poincaré algebra with standard commutators but deformed coproducts [126].

\section{DEFORMED DIFFEOMORPHISMS}

We first perform the Gaussian analysis for the derivation of brackets by defining $\star$-diffeomorphisms (or, equivalently, deformed diffeomorphisms) with a deformed action on single tensors but still respecting the Leibniz rule. This is done in an attempt to reproduce what has been studied for noncommutative quantum field theories [17-19,21,97-99], where the relevant $\star$-action is invariant under $\star-\mathrm{U}(1)$ symmetries obeying the Leibniz rule. Some rather encouraging results are achieved but we will explain later on why there is a strong reason for abandoning the Leibniz rule and then working with deformed diffeomorphisms with deformed comultiplication.

\section{A. Deformed diffeomorphisms with trivial coalgebra}

We define a deformed diffeomorphism by its infinitesimal action 


$$
\mathcal{L}_{v} \triangleright u:=v^{\rho} \star \partial_{\rho} \star u=v^{\rho} \star \partial_{\rho} u,
$$

on functions. In the last step we used the fact that, for the constant $\theta$ case, the action of the derivative is not modified, that is $\partial_{\mu} \star f \equiv \partial_{\mu} f$. Deformed diffeomorphisms are different from twisted ones because $v^{\rho} \star \partial_{\rho} u \neq \delta_{v} u$ defined in (41).

A deformed Gaussian system can be defined analogously to a twisted one. The first place where we used the Lie derivative in the construction of a twisted Gaussian system was in Eq. (47). Because it acts on a linear coordinate function $t$, it remains true if we use the Lie derivative (76) corresponding to deformed rather than twisted diffeomorphisms. The second place, the introduction of a condition on Gaussian vector fields, will be discussed soon. But first, we have to insert a warning about a violation of the standard Leibniz rule for the Lie derivative of deformed diffeomorphisms as defined so far.

In some sense, one could consider deformed diffeomorphisms the most natural definition of diffeomorphisms on $\mathcal{A}$. According to Eq. (23), we can obtain diffeomorphisms on $\mathcal{A}$ thanks to the mapping given by the $\star$-product. Using a Weyl map, we have

$\hat{\delta}_{V} F(\hat{x})=V(\hat{x}) \triangleright F(\hat{x}) \mapsto v(x) \star f(x)=v^{\rho}(x) \star \partial_{\rho} f(x)$,

where the last expression gives us exactly the definition we proposed for deformed diffeomorphisms, (76).

However, an extension to vector fields and tensors is nontrivial if we want to preserve the Leibniz rule. For instance, if we attempt such an extension by postulating that the $\star$-Lie derivative should agree with the Moyal bracket (38),

$$
\mathcal{L}_{v_{1}} \triangleright v_{2}=\left[v_{1}^{\star},_{2}\right],
$$

for two vector fields $v_{1}$ and $v_{2}$, the Leibniz rule is in danger when we apply the derivative to the product of a function $u$ and a vector field $w$ :

$$
\begin{aligned}
\mathcal{L}_{v} \triangleright(u \star w)= & v^{\rho} \star \partial_{\rho}\left(u \star w^{\mu}\right)-u \star w^{\rho} \star \partial_{\rho} v^{\mu} \\
= & \left(\mathcal{L}_{v} \triangleright u\right) \star w^{\mu}+u \star\left(\mathcal{L}_{v} \triangleright w\right) \\
& +\left(v^{\rho} \star u-u \star v^{\rho}\right) \star \partial_{\rho} w^{\mu} .
\end{aligned}
$$

The last $\star$-commutator violates the Leibniz rule, but it vanishes when $u$ is a constant, such as a normal component in our deformed Gaussian system. We may therefore postpone a detailed discussion of the Leibniz rule and first return to hypersurface deformations.

At this point, we have the necessary ingredients to develop the Gaussian analysis for deformed diffeomorphisms, with our general aim of deriving the hypersurfacedeformation brackets they imply. Due to the $\star$-modification of the action of these symmetries, it is then natural to expect modifications of the HDA and, thus, a deformed or $\star$-modification of general covariance.

\section{B. Modified Gaussian condition}

Recall that we are not interested in the Gaussian system in its own right, but rather have to make sure that the gauge choice leads to brackets of space-time vector fields which depend only on hypersurface data. The latter can then be reinterpreted as Lie-algebroid brackets. The original Gaussian condition for the metric reads

$$
n^{\mu} \star \mathcal{L}_{v} \star g_{\mu \nu}=0
$$

However, it does not lead to a well-defined Lie-algebroid structure for deformed diffeomorphisms. We modify it by subtracting a term which will lead to consistent relations:

$n^{\mu} \star \mathcal{L}_{v} \star g_{\mu \nu}-\partial_{\gamma}\left(v^{\rho} \star n^{\mu} \star g_{\rho \mu}\right) \star g^{\gamma \alpha} \star n^{\beta} \star g_{\alpha \beta} \star n_{\nu}=0$,

is the new $\star$-modified Gaussian condition. In abstract notation, the commutative analog of the new condition reads

$$
i_{n} \mathcal{L}_{v} g=\left(i_{n} \mathrm{~d}\left(i_{v} i_{n} g\right)\right) n,
$$

or, in components,

$$
n^{\mu} \mathcal{L}_{v} g_{\mu \nu}=n^{\rho} \partial_{\rho}\left(g_{\delta \gamma} n^{\gamma} v^{\delta}\right) n_{\nu}
$$

The difference with respect to the usual Gaussian condition is that the variation of the metric $g$ under a diffeomorphism along the direction identified by $v$ is nonzero. We are therefore choosing a different gauge where, instead of being zero, the normal contribution to $\mathcal{L}_{v} g$ is fixed to another specific value. Since the structure of hypersurface deformations should be gauge independent, we expect the new condition (82) to imply the same hypersurface-deformation brackets as derived in [95] when applied to the ordinary product. In the Appendix, we confirm that this modification indeed does not change the result of the classical calculation for commutative theories. As a brief argument, we can see that the classical condition can be modified by our counterterm because the latter is zero when the conditions for lapse and shift that follow from the original condition are satisfied, in particular when $0=n^{\rho} \partial_{\rho}\left(N^{2}\right)=i_{n} \mathrm{~d}\left(i_{v} i_{n} g\right)$. (The counterterm vanishes "on shell").

Using the Cartan identity, we write the modified Gaussian condition as

$$
\begin{gathered}
i_{v} \star \mathrm{d} n+\mathrm{d}\left(i_{v} \star i_{n} \star g\right)+i_{[n * v]} \star g \\
+\left(\mathrm{d}\left(i_{v} \star i_{n} \star g\right) \star \overleftarrow{i_{n}}\right) \star n=0,
\end{gathered}
$$

where $\overleftarrow{i_{n}}$ highlights the fact that the normal vector is $\star$-contracted with the tensor on the left of the product, 
$\mathrm{d}\left(i_{v} \star i_{n} \star g\right)$. Decomposing $v=N \star n+M \star X$ and using $\mathrm{d} n=0$ as well as the orthogonality conditions

$$
i_{n} \star g \star i_{n}=-1 \quad i_{X} \star i_{n} \star g=0,
$$

we find

$$
\left[n^{\star} N \star n\right] \star g+[n, M \star X] \star g=\mathrm{d} N+\left(\mathrm{d} N \star i_{n}\right) \star n
$$

or

$i_{n} \star \mathrm{d} N \star n^{\mu} \star g_{\mu \nu}+[n \star M \star X]^{\mu} \star g_{\mu \nu}=\partial_{\nu} N+\partial_{\gamma} N \star n^{\gamma} \star n_{\nu}$.

We extract the tangential part by $\star$-multiplying both sides of the equation by $g^{\nu \alpha} \star q_{\alpha}^{a}$ from the right

$$
\left[n^{\star}, M \star X\right]^{a}=\partial_{\nu} N \star g^{\nu \alpha} \star q_{\alpha}^{a},
$$

and the normal part by $\star$-multiplying both sides of the equation by $n^{\nu}$ from the right

$$
-n^{\rho} \star \partial_{\rho} N+\left[n^{\star}, M \star X\right]^{\mu} \star g_{\mu \nu} \star n^{\nu}=0 .
$$

The commutator term is equal to

$$
\begin{aligned}
{\left[n^{\star}, M \star X\right]^{\mu} \star g_{\mu \nu} \star n^{\nu}=} & n^{\rho} \star \partial_{\rho}(M \star X)^{\mu} \star g_{\mu \nu} \star n^{\nu} \\
& -(M \star X)^{\rho} \star\left(\partial_{\rho} n^{\mu}\right) \star g_{\mu \nu} \star n^{\nu} .
\end{aligned}
$$

In our Gaussian frame, $n^{\alpha}$ is normalized, geodesic, and has constant components. The commutator is therefore zero and we have

$$
n^{\nu} \star \partial_{\nu} N=0
$$

Since the components $n^{\nu}$ are constant, the $\star$-product does not imply higher derivatives in this equation. Therefore, we still have a well-posed initial-value problem for lapse $N$ and shift $M^{a}$.

Using a decomposition as in (73), we now obtain

$$
\left[\left(N_{1}, 0\right) \star\left(N_{2}, 0\right)\right]=\left(0,\left(N_{1} \star \partial_{b} N_{2}-N_{2} \star \partial_{b} N_{1}\right) \star q^{a b}\right) .
$$

For brackets involving tangential vector fields, we have

$$
\left[\left(0, M_{1}^{a}\right)^{\star},\left(0, M_{2}^{a}\right)\right]=\left(0,\left[M_{1} \star X^{\star}, M_{2} \star X\right]^{\alpha} \star q_{\alpha}^{a}\right)
$$

and

$$
\left[(N, 0){ }^{\star}\left(0, M^{a}\right)\right]=\left(-\mathcal{L}_{M \star X} \triangleright N, 0\right) .
$$

Therefore, we are able to derive a well-defined HDA in our modified Gaussian frame. It has the form of the classical version without any correction term other than a generalization to Moyal space. This means that we find for the $\star$-HDA the same form of the classical HDA but with the usual point product replaced by the $\star$-product. Note, however, that the $\star$-product implies higher time derivatives which affect the interpretation of the HDA. We will comment on this implication in more detail in Sec. IV D.

We now have a possible candidate for a $\star$-HDA. According to Ref. [17], for instance, once the deformation of infinitesimal diffeomorphisms has been introduced, the action for gravity should be written with the requirement of invariance under these $\star$-symmetries. In particular, the deformed Einstein-Hilbert action should be formulated in terms of star-products. However, in order to make sure that there is a fully covariant tensor calculus, we have to return to a discussion of the Leibniz rule.

\section{Modified Leibniz rule}

The demonstration that an action for noncommutative gravity, such as the one introduced in Ref. [87], is covariant requires an application of the Leibniz rule. In particular, inserting the Lie derivative $\delta_{v} L$ in the Lagrangian density $L=E \star R$ in an action

$$
S_{\star}=\int \mathrm{d}^{4} x L=\int \mathrm{d}^{4} x E \star R,
$$

where $\mathrm{d}^{4} x E$ is a suitably deformed measure and $R$ is the $\star$-Ricci scalar, should result in a boundary term. (See, for instance, [87] for details and explicit expressions.)

Assuming the Leibniz rule, the infinitesimal variation of the Lagrangian density under deformed diffeomorphisms would be given by

$$
\begin{aligned}
\delta_{v} L & =\mathcal{L}_{v} \triangleright(E \star R)=\left(\mathcal{L}_{v} \triangleright E\right) \star R+E \star \mathcal{L}_{v} \triangleright R \\
& =\left(v^{\rho} \star \partial_{\rho} E+\partial_{\rho} v^{\rho} \star E\right) \star R+E \star v^{\rho} \star \partial_{\rho} R \\
& =\partial_{\rho}\left(v^{\rho} \star E \star R\right)+E \star v^{\rho} \star \partial_{\rho} R-v^{\rho} \star E \star \partial_{\rho} R,
\end{aligned}
$$

which differs from a total derivative by the nonzero star commutator $\left(E \star v^{\rho}-v^{\rho} \star E\right) \star \partial_{\rho} R$. However, foregoing the Leibniz rule at this point and applying the Lie derivative directly to the density $E \star R$ does give us a total derivative:

$$
\begin{aligned}
\mathcal{L}_{v} \triangleright(E \star R) & =v^{\rho} \star \partial_{\rho}(E \star R)+\left(\partial_{\rho} v^{\rho} \star\right)(E \star R) \\
& =\partial_{\rho}\left(v^{\rho} \star E \star R\right) .
\end{aligned}
$$

The action would then be invariant but the Lie derivative does not agree with (95).

We therefore have to refine our notion of deformed diffeomorphisms, in contrast to the situation in noncommutative field theories [17,18], for which there are $\star$-actions invariant under both twisted $\mathrm{U}(N)$ transformations with non-trivial coproducts and deformed $\mathrm{U}(N)$ 
transformations with standard Leibniz rule [21]. The main reason why we tried to define $\star$-diffeomorphisms with trivial co-multiplication was the desire to mimic what happens in noncommutative quantum field theories, but we now see that there is a pronounced difference between noncommutative gravity and other noncommutative systems at a fundamental level.

In our example of a density times the Ricci scalar, the defect in the Leibniz rule was given by a star commutator of components. We can therefore try to modify the Leibniz rule by rearranging different factors. We now define

$\mathcal{L}_{v} \triangleright(u \star w):=\left(\mathcal{L}_{v} \triangleright u\right) \star w+\bar{R}(u) \star\left(\mathcal{L}_{\bar{R}(v)} \triangleright w\right)$,

where $\bar{R}$ is defined in (30). Together with this deformed Leibniz rule, we also change the ordering in the action of $\star$-diffeomorphisms on vectors to obtain the new Lie derivative

$$
\mathcal{L}_{v} \triangleright u^{\mu}:=v^{\rho} \star \partial_{\rho} u^{\mu}-\partial_{\rho} v^{\mu} \star u^{\rho} .
$$

Now we can prove that $u^{\mu} \star u_{\mu}$ transforms as a scalar under deformed diffeomorphisms: We have

$$
\begin{aligned}
\left(\mathcal{L}_{v} \triangleright u^{\mu}\right) \star u_{\mu}+\bar{R}\left(u^{\mu}\right) \star\left(\mathcal{L}_{\bar{R}(v)} \triangleright u_{\mu}\right) \\
=\left(v^{\rho} \star \partial_{\rho} u^{\mu}-\partial_{\rho} v^{\mu} \star u^{\rho}\right) \star u_{\mu}+\bar{R}\left(u^{\mu}\right) \star\left(\bar{R}\left(v^{\rho}\right) \star \partial_{\rho} u_{\mu}\right. \\
\left.\quad+\bar{R}\left(\partial_{\mu} v^{\rho}\right) \star u_{\rho}\right)=v^{\rho} \star \partial_{\rho} u^{\mu} \star u_{\mu} \\
\quad-\partial_{\rho} v^{\mu} \star u^{\rho} \star u_{\mu}+v^{\rho} \star u^{\mu} \star \partial_{\rho} u_{\mu}+\partial_{\mu} v^{\rho} \star u^{\mu} \star u_{\rho} .
\end{aligned}
$$

The second and the fourth terms in the last line cancel out, and we have

$$
\begin{aligned}
& \left(\mathcal{L}_{v} \triangleright u^{\mu}\right) \star u_{\mu}+\bar{R}\left(u^{\mu}\right) \star\left(\mathcal{L}_{\bar{R}(v)} \triangleright u_{\mu}\right) \\
& =v^{\rho} \star \partial_{\rho} u^{\mu} \star u_{\mu}+v^{\rho} \star u^{\mu} \star \partial_{\rho} u_{\mu}=\mathcal{L}_{v} \triangleright\left(u^{\mu} \star u_{\mu}\right) .
\end{aligned}
$$

In order to prove that the new Leibniz rule implies a consistent extension of the deformed Lie derivative to tensors, we start with the $\star$ product of two vector fields, $u_{1}^{\mu} \star u_{2}^{\nu}$ :

$$
\begin{aligned}
& \mathcal{L}_{v} \triangleright\left(u_{1}^{\mu} \star u_{2}^{\nu}\right)=\left(\mathcal{L}_{v} \triangleright u_{1}^{\mu}\right) \star u_{2}^{\nu}+\bar{R}\left(u_{1}^{\mu}\right) \star\left(\mathcal{L}_{\bar{R}(v)} \triangleright u_{2}^{\nu}\right) \\
& =v^{\rho} \star \partial_{\rho} u_{1}^{\mu} \star u_{2}^{\nu}-\partial_{\rho} v^{\mu} \star u_{1}^{\rho} \star u_{2}^{\nu} \\
& +v^{\rho} \star u_{1}^{\mu} \star \partial_{\rho} u_{2}^{\nu}-\partial_{\rho} v^{\nu} \star u_{1}^{\mu} \star u_{2}^{\rho} \\
& =v^{\rho} \star \partial_{\rho}\left(u_{1}^{\mu} \star u_{2}^{\nu}\right)-\partial_{\rho} v^{\mu} \star u_{1}^{\rho} \star u_{2}^{\nu} \\
& -\partial_{\rho} v^{\nu} \star u_{1}^{\mu} \star u_{2}^{\rho}=\mathcal{L}_{v} \triangleright T^{\mu \nu}
\end{aligned}
$$

with the contravariant 2-tensor $T^{\mu \nu}:=u_{1}^{\mu} \star u_{2}^{\nu}$. By induction, the claim then follows for arbitrary tensors:

$$
\begin{aligned}
& \mathcal{L}_{v} \triangleright\left(u_{1}^{\mu_{1}} \star u_{2}^{\mu_{2}} \star \ldots \star u_{n}^{\mu_{n}} \star w_{\nu_{1}}^{1} \star \ldots \star w_{\nu_{n}}^{n}\right) \\
& =\left(\mathcal{L}_{v} \triangleright u_{1}^{\mu_{1}}\right) \star\left(u_{2}^{\mu_{2}} \star \ldots \star u_{n}^{\mu_{n}} \star w_{\nu_{1}}^{1} \star \ldots \star w_{\nu_{n}}^{n}\right) \\
& +\bar{R}_{1}\left(u_{1}^{\mu_{1}}\right) \star\left(\mathcal{L}_{\bar{R}_{1}(v)} \triangleright\left(u_{2}^{\mu_{2}} \star \ldots \star u_{n}^{\mu_{n}} \star w_{\nu_{1}}^{1} \star \ldots \star w_{\nu_{n}}^{n}\right)\right) \\
& =\left(v^{\rho} \star \partial_{\rho} u_{1}^{\mu_{1}}\right) \star\left(u_{2}^{\mu_{2}} \star \ldots \star u_{n}^{\mu_{n}} \star w_{\nu_{1}}^{1} \star \ldots \star w_{\nu_{n}}^{n}\right) \\
& -\left(\partial_{\rho} v^{\mu_{1}} \star u_{1}^{\rho}\right) \star\left(u_{2}^{\mu_{2}} \star \ldots \star u_{n}^{\mu_{n}} \star w_{\nu_{1}}^{1} \star \ldots \star w_{\nu_{n}}^{n}\right) \\
& +v^{\rho} \star u^{\mu_{1}} \star \partial_{\rho} u_{2}^{\mu_{2}} \star u_{3}^{\mu_{3}} \star \ldots \star u_{n}^{\mu_{n}} \star w_{\nu_{1}}^{1} \star \ldots \star w_{\nu_{n}}^{n} \\
& -\partial_{\rho} v^{\mu_{2}} \star u_{1}^{\mu_{1}} \star u_{2}^{\rho} \star u_{3}^{\mu_{3}} \star \ldots \star u_{n}^{\mu_{n}} \star w_{\nu_{1}}^{1} \star \ldots \star w_{\nu_{n}}^{n} \\
& +\bar{R}_{1}\left(u_{1}^{\mu_{1}}\right) \star \bar{R}_{2}\left(u_{2}^{\mu_{2}}\right) \star\left(\mathcal { L } _ { \overline { R } _ { 2 } \overline { R } _ { 1 } ( v ) } \triangleright \left(u_{3}^{\mu_{3}} \star \ldots \star u_{n}^{\mu_{n}}\right.\right. \\
& \left.\left.\star w_{\nu_{1}}^{1} \star \ldots \star w_{\nu_{n}}^{n}\right)\right)=\cdots=\mathcal{L}_{v} \triangleright\left(T_{\nu_{1} \nu_{2} \ldots \nu_{n}}^{\mu_{1} \mu_{2} \ldots \mu_{n}}\right)
\end{aligned}
$$

with $T_{\nu_{1} \nu_{2} \ldots \nu_{n}}^{\mu_{1} \mu_{2} \ldots \mu_{n}}:=u_{1}^{\mu_{1}} \star u_{2}^{\mu_{2}} \star \ldots \star u_{n}^{\mu_{n}} \star w_{\nu_{1}}^{1} \star \ldots \star w_{\nu_{n}}^{n}$.

\section{Deformed diffeomorphisms with deformed Leibniz rule}

We have clarified the reason why the Leibniz rule has to be modified when we adopt a noncommutative multiplication rule, and provided a new definition to resolve the problem. With this result, we can now focus on the derivation of the hypersurface-deformation brackets for deformed diffeomorphisms with deformed Leibniz rule as in Eq. (97).

Combining the lessons from our previous derivation with the standard Leibniz rule as well as the new Lie derivative, we now introduce a modified Gaussian condition by requiring

$$
\begin{aligned}
& \bar{R}\left(n^{\mu}\right) \star\left(\mathcal{L}_{\bar{R}(v)} \triangleright g_{\mu \nu}\right) \\
& \quad=-\partial_{\rho}\left(v^{\rho} \star n^{\nu} \star g_{\mu \rho}\right) \star n^{\gamma} \star n^{\rho} \star g_{\gamma \nu}
\end{aligned}
$$

for space-time vector fields $v$. Using the modified Leibniz rule we can rewrite this equation as

$$
\begin{aligned}
\mathcal{L}_{v} & \triangleright\left(n^{\mu} \star g_{\mu \nu}\right)-\left(\mathcal{L}_{v} \triangleright n^{\mu}\right) \star g_{\mu \nu} \\
& =-\partial_{\rho}\left(v^{\rho} \star n^{\nu} \star g_{\mu \rho}\right) \star n^{\gamma} \star n^{\rho} \star g_{\gamma \nu},
\end{aligned}
$$

and thanks to the Cartan identity, obtain

$$
\begin{gathered}
\partial_{\nu}\left(v^{\rho} \star n^{\mu} \star g_{\mu \rho}\right)+v^{\rho} \star(\mathrm{d} n)_{\rho \nu}-[v, n]_{\star}^{\mu} \star g_{\mu \nu} \\
=-\partial_{\rho}\left(v^{\rho} \star n^{\nu} \star g_{\mu \rho}\right) \star n^{\gamma} \star n^{\rho} \star g_{\gamma \nu} .
\end{gathered}
$$

Here $(\mathrm{d} n)_{\rho \nu} \equiv \partial_{\rho}\left(n^{\mu} \star g_{\mu \nu}\right)-\partial_{\nu}\left(n^{\mu} \star g_{\mu \rho}\right) \quad$ vanishes as before. Decomposing $v^{\mu}=N \star n^{\mu}+M^{a} \star X_{a}^{\mu}$, we find

$$
\begin{aligned}
& -\partial_{\nu} N-[N \star n, n]_{\star}^{\mu} \star g_{\mu \nu}-[M \star X, n]_{\star}^{\mu} \star g_{\mu \nu} \\
& =\partial_{\rho} N \star n^{\gamma} \star n^{\rho} \star g_{\gamma \nu} .
\end{aligned}
$$


Projection implies the normal part

$$
\begin{aligned}
& -\partial_{\nu} N \star g^{\nu \alpha} \star n^{\beta} \star g_{\alpha \beta}-[N \star n, n]_{\star}^{\alpha} \star n^{\beta} \star g_{\alpha \beta} \\
& -[M \star X, n]_{\star}^{\alpha} \star n^{\beta} \star g_{\alpha \beta} \\
& =\partial_{\rho} N \star n^{\gamma} \star n^{\rho} \star g_{\gamma \nu} \star g^{\nu \alpha} \star n^{\beta} \star g_{\alpha \beta},
\end{aligned}
$$

or

$$
\begin{aligned}
& -\partial_{\nu} N \star n^{\nu}-N \star n^{\rho} \star \partial_{\rho} n^{\alpha} \star n^{\beta} \star g_{\alpha \beta} \\
& +\partial_{\rho}\left(N \star n^{\alpha}\right) \star n^{\rho} \star n^{\beta} \star g_{\alpha \beta} \\
& -[M \star X, n]_{\star}^{\alpha} \star n^{\beta} \star g_{\alpha \beta} \\
& =\partial_{\rho} N \star n^{\alpha} \star n^{\rho} \star n^{\beta} \star g_{\alpha \beta} .
\end{aligned}
$$

We now use $n^{\rho} \star \partial_{\rho} n^{\mu}=0$, cancel out $\partial_{\rho} N \star n^{\alpha} \star n^{\rho} \star n^{\beta} \star g_{\alpha \beta}$, and obtain

$$
-\partial_{\nu} N \star n^{\nu}=[M \star X, n]_{\star}^{\alpha} \star n^{\beta} \star g_{\alpha \beta} .
$$

The commutator on the right is equal to

$$
\begin{aligned}
{[M \star X, n]_{\star}^{\alpha} \star n^{\beta} \star g_{\alpha \beta} } & =(M \star X)^{\gamma} \star\left(\partial_{\gamma} n^{\alpha}\right) \star n^{\beta} \star g_{\alpha \beta} \\
& -n^{\gamma} \star \partial_{\gamma}(M \star X)^{\alpha} \star n^{\beta} \star g_{\alpha \beta} .
\end{aligned}
$$

If we now use the properties of our Gaussian frame, in particular that $n^{\alpha}$ is normalized, geodesic, and has constant components, the commutator is zero and we arrive at

$$
-\partial_{\nu} N \star n^{\nu}=0
$$

The tangential part of (104) is

$$
\begin{aligned}
& -\partial_{\nu} N \star g^{\nu \alpha} \star q_{\alpha b}-[M \star X, n]_{\star}^{\alpha} \star q_{\alpha b}-[N \star n, n]_{\star}^{\alpha} \star q_{\alpha b} \\
& =-\partial_{b} N-[M \star X, n]_{\star}^{\alpha} \star q_{\alpha b}-N \star n^{\rho} \star \partial_{\rho} n^{\alpha} \star q_{\alpha b} \\
& -\partial_{\rho} N \star n^{\alpha} \star n^{\rho} \star q_{\alpha b} \\
& =\partial_{\rho} N \star n^{\alpha} \star n^{\rho} \star q_{\alpha b},
\end{aligned}
$$

which is equivalent to

$$
[M \star X, n]_{\star}^{a}=-\partial_{b} N \star q^{a b} .
$$

As before, the equations for lapse and shift provide a wellposed initial-value problem.

We can now compute the bracket

$$
\begin{aligned}
{\left[v_{1}, v_{2}\right]_{\star}^{\mu}=} & {\left[N_{1} \star n, N_{2} \star n\right]_{\star}^{\mu}+\left[N_{1} \star n, M_{2} \star X\right]_{\star}^{\mu} } \\
& +\left[M_{1} \star X, N_{2} \star n\right]_{\star}^{\mu}+\left[M_{1} \star X, M_{2} \star X\right]_{\star}^{\mu} \\
= & N_{1} \star n^{\rho} \star \partial_{\rho}\left(N_{2} \star n^{\mu}\right)-\partial_{\rho}\left(N_{1} \star n^{\mu}\right) \star N_{2} \star n^{\rho} \\
& +N_{1} \star n^{\rho} \star \partial_{\rho}\left(M_{2} \star X^{\mu}\right) \\
& -\partial_{\rho}\left(N_{1} \star n^{\mu}\right) \star M_{2} \star X^{\rho} \\
& +M_{1} \star X^{\rho} \star \partial_{\rho}\left(N_{2} \star n^{\mu}\right) \\
& -\partial_{\rho}\left(M_{1} \star X^{\mu}\right) \star N_{2} \star n^{\rho} \\
& +\left[M_{1} \star X, M_{2} \star X\right]_{\star}^{b} \star X_{b}^{a} .
\end{aligned}
$$

Choosing Gaussian vector fields with either zero lapse $N$ or shift $M^{a}$ functions we can decompose the above brackets as a set of three distinct commutators $\left[\left(0, M_{1}\right),\left(0, M_{2}\right)\right]_{\star}$, $\left[\left(0, M_{1}\right),\left(N_{2}, 0\right)\right]_{\star}$ and $\left[\left(N_{1}, 0\right),\left(N_{2}, 0\right)\right]_{\star}$. If both lapse functions are zero, we find

$$
\left[\left(0, M_{1}\right),\left(0, M_{2}\right)\right]_{\star}=\left(0,\left[M_{1} \star X, M_{2} \star X\right]_{\star}^{a}\right) .
$$

For both shift vector fields equal to zero, we obtain

$$
\left[\left(N_{1}, 0\right),\left(N_{2}, 0\right)\right]_{\star}=\left(0, N_{1} \star q^{a b} \star \partial_{b} N_{2}-\partial_{b} N_{1} \star N_{2} \star q^{a b}\right) .
$$

The remaining bracket reads

$$
[(0, M),(N, 0)]_{\star}=\left(\mathcal{L}_{M \star X} \triangleright N, 0\right) .
$$

It is perhaps surprising that the overall structure of the bracket between $N$ and $M^{a}$ is preserved despite the noncommutativity of coordinates. In this regard, one can note that the $\star$-Lie bracket between two tangential deformations still gives us a tangential hypersurface deformation, the one involving a normal and a tangential deformations gives a normal displacement, and the bracket between two normal deformations results in a spatial shift. The only type of modifications that appear with respect to the standard hypersurface brackets are higher derivative terms. Those terms are implicit in the above expressions, but it is clear that such terms appear as soon as we expand the Moyal star product by powers of $\theta$.

Although the brackets bear a formal resemblance with the classical ones, their detailed form is markedly different. The main reason is the nonlocality of the $\star$-product, which includes higher derivatives in space-time. In the noncommutative HDA brackets as written, we therefore have time derivatives of $N, M^{a}$ and the inverse spatial metric $q^{a b}$, which, unlike those of the constant $n^{\mu}$, are in general nonzero. Since the brackets cannot contain space-time data, we should interpret the $\star$-products in them as follows: Working in the Gaussian frame, time derivatives of $N$ and $M^{a}$ can be replaced by spatial derivatives using the Eqs. (107) and (108). Any first-order time derivative of $q_{a b}$ can be expressed as a linear combination of extrinsic-curvature components $K_{a b}$, while higher-order time derivatives of $q_{a b}$ are related to higher-order momenta in the Ostrogradsky treatment of a canonical higherderivative theory. Without a specific noncommutative action, we cannot write these terms explicitly, but rather leave the brackets in the form (110) with implicit higherderivative terms.

We conclude that the base manifold of the noncommutative HDA should contain not only the spatial metric but the entire phase space of a higher-derivative metric theory. The presence of extrinsic curvature among these variables is reminiscent of holonomy modifications in models of 
loop quantum gravity, but the explicit dependence is, in general, different (see e.g., [105,106]).

\section{CONCLUSIONS}

We have studied infinitesimal diffeomorphisms on noncommutative manifolds equipped with a nonstandard multiplication rule in terms of $\star$-products. Previous studies on noncommutative formulations of gravity (in particular $[87,88])$ succeeded in twisting the group of 4-dimensional diffeomorphisms, thereby achieving a deformation of GR symmetries in the sense of Drinfeld twists [40,41]. Nonetheless, as already pointed out in the literature and further stressed in this work, it remains unclear whether diffeomorphisms on noncommutative spaces should be introduced by means of twisting or explicitly deforming their action, as it is the case for $\star$-gauge transformations in noncommutative extensions of quantum field theories.

The study of the algebra of hypersurface deformations, generating diffeomorphisms if we make a $3+1$ splitting of the 4-manifold, can provide additional insights into general covariance in noncommutative gravity as well as on the counting of physical d.o.f. of the theory. Our analysis is one of only a few in the context of canonical formulations of noncommutative gravity. In addition to shedding some light on long-standing questions in noncommutative gravity, it might also help in making contact with other recently proposed modifications of the HDA [105-112]. One possible point of contact is the presence of extrinsic curvature as one of the coordinates on the base manifold of a noncommutative HDA.

By using a recently developed approach to the derivation of the HDA [95], we have shown a constructive method to derive the brackets between spatial and time components of Gaussian vector fields when functions and tensors are multiplied with a noncommutative $\star$-product. This application is conceptually different from the derivation in classical general relativity given in [95] because we cannot take for granted that there is a covariant theory with a welldefined HDA on noncommutative manifolds. We therefore had to demonstrate that the frame of a Gaussian system, used in [95], can be suitably generalized to specific types of noncommutativity. After doing this, we derive well-defined HDAs, which implies that there are infinitesimal space-time transformations that allow us to change the frame. In this sense, we have demonstrated the covariance of such theories, even though we did not use an explicit action principle.

In particular, we have studied both the HDA encoding twisted diffeomorphisms and the deformations of the HDA produced by what we call deformed or $\star$-diffeomorphisms. In the former case, we have found, not surprisingly, that the brackets are unmodified compared with the classical algebra of GR gravitational constraints. This result confirms some of the previous statements that appeared in the literature on twisted gravity [87].
In the analysis of the latter case-deformed diffeomorphisms - we did not have any guidance from established results. Thus, building on the analogy with $\star-\mathrm{U}(1)$ [or in general $\star-\mathrm{U}(N)$ ] gauge theories, we first defined deformed diffeomorphisms with a suitably deformed action on single fields but retaining the Leibniz rule in their action on the $\star$-product of two or more functions. We were able to overcome the technical challenges represented by the correction terms to the HDA brackets, but eventually recognized a major obstacle to the implementation of a consistent noncommutative differential calculus where diffeomorphism transformations have a trivial coalgebra. This forced us to deform the coproducts of $\star$-diffeomorphisms. As a result, we have reached a meaningful deformation of the HDA for deformed diffeomorphisms without pathological correction terms and with a consistent differential calculus suitably adapted to $\star$-products.

While formally similar to the classical HDA, noncommutative HDAs based on deformed diffeomorphisms show crucial differences in their structure owing to nonlocality (in particular in time) of $\star$-products. We hope that this result may serve as a base for an alternative formulation of noncommutative gravity in terms of the deformed diffeomorphisms put forward here, instead of relying on the symmetry principle of twisting as done so far. The path we followed here provides a simplified way to get insight into how general covariance might be affected by $\star$-products or other possible deformations.

For twisted diffeomorphisms we have also been able to discuss straightforwardly the flat-spacetime (or Minkowski) limit since we had no deformations of the HDA. On the contrary, the study of the Minkowski regime of the deformed HDA encoding $\star$-diffeomorphisms remains an open challenge which should be of particular interest both from the perspective of relating $\star$-product corrections to the nonlinear Poincaré transformations of noncommutative spacetimes $[67,68]$ and also to have a better understanding of what general modifications of the HDA should affect the Poincaré algebra.

\section{ACKNOWLEDGMENTS}

This work was supported in part by NSF Grant No. PHY1607414. M. R. thanks the Institute for Gravitation and the Cosmos and Penn State University for the hospitality during the elaboration of this project. The work of M. R. is supported by a mobility grant awarded by Sapienza University of Rome and MIUR. The contribution of M. R. is based upon work from COST Action MP1405 QSPACE, supported by COST (European Cooperation in Science and Technology).

\section{APPENDIX: MODIFIED GAUSSIAN CONDITION: CLASSICAL CASE}

In this Appendix, we show that a suitable modification (82) of the Gaussian system still results in the usual classical HDA. We begin with 


$$
n^{\mu} \mathcal{L}_{v} g_{\mu \nu}=n^{\rho} \partial_{\rho}\left(g_{\delta \gamma} n^{\gamma} v^{\delta}\right) n_{\nu}
$$

and write the Lie derivative explicitly:

$n^{\mu} v^{\sigma} \partial_{\sigma} g_{\mu \nu}+n^{\mu} g_{\mu \sigma} \partial_{\nu} v^{\sigma}+n^{\mu} g_{\sigma \nu} \partial_{\mu} v^{\sigma}=n^{\rho} \partial_{\rho}\left(g_{\delta \gamma} n^{\gamma} v^{\delta}\right) n_{\nu}$,

or, equivalently,

$$
\begin{aligned}
\partial_{\nu} & \left(g_{\mu \sigma} n^{\mu} v^{\sigma}\right)-v^{\sigma} \partial_{\nu}\left(g_{\mu \sigma} n^{\mu}\right)+v^{\sigma} \partial_{\sigma}\left(g_{\mu \nu} n^{\mu}\right) \\
& -v^{\sigma} g_{\mu \nu} \partial_{\sigma} n^{\mu}+n^{\mu} g_{\sigma \nu} \partial_{\mu} v^{\sigma} \\
= & n^{\rho} \partial_{\rho}\left(g_{\delta \gamma} n^{\gamma} v^{\delta}\right) n_{\nu} .
\end{aligned}
$$

Using $(\mathrm{d} n)_{\sigma \nu}:=\partial_{\sigma}\left(g_{\mu \nu} n^{\mu}\right)-\partial_{\nu}\left(g_{\mu \sigma} n^{\mu}\right) \quad$ and $\quad[n, v]^{\mu}=$ $n^{\rho} \partial_{\rho} v^{\mu}-v^{\rho} \partial_{\rho} n^{\mu}$, we obtain

$\partial_{\nu}\left(g_{\mu \sigma} n^{\mu} v^{\sigma}\right)+v^{\sigma}(\mathrm{d} n)_{\sigma \nu}+[n, v]^{\mu} g_{\mu \nu}=n^{\rho} \partial_{\rho}\left(g_{\delta \gamma} n^{\gamma} v^{\delta}\right) n_{\nu}$.

If we choose the metric such that

$$
\mathrm{d} s^{2}=-\mathrm{d} t^{2}+q_{a b} \mathrm{~d} x^{a} \mathrm{~d} x^{b}
$$

we have $\mathrm{d} n=\mathrm{d}^{2} t=0$. Although our Gaussian condition has been modified, the metric (A3) is consistent with the gauge choice as shown by the final result, in particular Eq. (A7). Moreover,

$$
\partial_{\nu}\left(g_{\mu \sigma} n^{\mu} v^{\sigma}\right)+[n, v]^{\mu} g_{\mu \nu}=n^{\rho} \partial_{\rho}\left(g_{\delta \gamma} n^{\gamma} v^{\delta}\right) n_{\nu} .
$$

Decomposing $v$ as $v^{\mu}=N n^{\mu}+M^{\mu}$ we obtain

$$
-\partial_{\nu} N+[n, M]^{\mu} g_{\mu \nu}=-n^{\rho} \partial_{\rho} N n_{\nu} .
$$

Let us now find the normal and tangential components of the above equality. For the normal we have

$$
-n^{\nu} \partial_{\nu} N+[n, M]^{\nu} n_{\nu}=n^{\rho} \partial_{\rho} N
$$

and thus

$$
n^{\nu} \partial_{\nu} N=0
$$

because $[n, M]$ does not have a normal component. For the tangential part, we obtain

$$
[n, M]^{a}=q^{a b} \partial_{b} N
$$

We are now ready to compute the bracket between two vector fields:

$$
\begin{aligned}
{\left[v_{1}, v_{2}\right]^{\mu}=} & \left(N_{1} \mathcal{L}_{n} N_{2}-N_{2} \mathcal{L}_{n} N_{1}+\mathcal{L}_{M_{1}} N_{2}-\mathcal{L}_{M_{2}} N_{1}\right) n^{\mu} \\
& -N_{2}\left[n, M_{1}\right]+N_{1}\left[n, M_{2}\right]+\left[M_{1}, M_{2}\right]^{\mu} \\
= & \left(N_{1} n^{\rho} \partial_{\rho} N_{2}-N_{2} n^{\rho} \partial_{\rho} N_{1}+M_{1}^{b} \partial_{b} N_{2}\right. \\
& \left.-M_{2}^{b} \partial_{b} N_{1}\right) n^{\mu} \\
& +N_{1} q^{a b} \partial_{b} N_{2}-N_{2} q^{a b} \partial_{b} N_{1}+\left[M_{1}, M_{2}\right]^{a} \\
= & \left(M_{1}^{b} \partial_{b} N_{2}-M_{2}^{b} \partial_{b} N_{1}\right) n^{\mu} \\
& +q^{a b}\left(N_{1} \partial_{b} N_{2}-N_{2} \partial_{b} N_{1}\right)
\end{aligned}
$$

where we used Eqs. (A7) and (A8). Finally, we can extract the normal and tangential components of the brackets:

$$
\begin{gathered}
{\left[\left(0, M_{1}\right),\left(0, M_{2}\right)\right]=\left(0, \mathcal{L}_{M_{1}} M_{2}\right)} \\
{\left[\left(N_{1}, 0\right),\left(0, M_{2}\right)\right]=-\left(\mathcal{L}_{M_{2}} N_{1}, 0\right)}
\end{gathered}
$$

$\left[\left(N_{1}, 0\right),\left(N_{2}, 0\right)\right]=\left(0, q^{a b}\left(N_{1} \partial_{b} N_{2}-N_{2} \partial_{b} N_{1}\right)\right)$.

These are the brackets of Dirac's hypersurface-deformation algebroid.
[1] S. Doplicher, K. Fredenhagen, and J.E. Roberts, The quantum structure of space-time at the Planck scale and quantum fields, Commun. Math. Phys. 172, 187 (1995).

[2] S. Doplicher, K. Fredenhagen, and J. E. Roberts, Spacetime quantization induced by classical gravity, Phys. Lett. B 331, 39 (1994).

[3] G. Veneziano, A stringy nature needs just two constants, Europhys. Lett. 2, 199 (1986).

[4] T. Padmanabhan, Limitations on the operational definition of spacetime events and quantum gravity, Classical Quantum Gravity 4, L107 (1987).

[5] K. Konishi, G. Paffuti, and P. Provero, Minimum physical length and the generalized uncertainty principle in string theory, Phys. Lett. B 234, 276 (1990).
[6] J. Ellis, N. E. Mavromatos, and D. V. Nanopoulos, String theory modifies quantum mechanics, Phys. Lett. B 293, 37 (1992).

[7] L. J. Garay, Quantum gravity and minimum length, Int. J. Mod. Phys. A 10, 145 (1995).

[8] D. V. Ahluwalia, Quantum measurements, gravitation, and locality, Phys. Lett. B 339, 301 (1994).

[9] Y. J. Ng and H. Van Dam, Limit to space-time measurement, Mod. Phys. Lett. A 09, 335 (1994).

[10] G. Amelino-Camelia, Limits on the measurability of space-time distances in the semiclassical approximation of quantum gravity, Mod. Phys. Lett. A 09, 3415 (1994).

[11] A. Kempf, G. Mangano, and R. B. Mann, Hilbert space representation of the minimal length uncertainty relation, Phys. Rev. D 52, 1108 (1995). 
[12] M. Maggiore, A generalized uncertainty principle in quantum gravity, Phys. Lett. B 304, 65 (1993).

[13] G. Amelino-Camelia, G. Calcagni, and M. Ronco, Imprint of quantum gravity in the dimension and fabric of spacetime, Phys. Lett. B 774, 630 (2017).

[14] G. Calcagni and M. Ronco, Dimensional flow and fuzziness in quantum gravity: Emergence of stochastic spacetime, Nucl. Phys. B923, 144 (2017).

[15] A. Connes, Geometry and the quantum, arXiv:1703 .02470 .

[16] A. Connes, M. R. Douglas, and A. S. Schwarz, Noncommutative geometry and matrix theory: Compactification on tori, J. High Energy Phys. 02 (1998) 003.

[17] R. J. Szabo, Quantum field theory on noncommutative spaces, Phys. Rep. 378, 207 (2003).

[18] J. Ambjorn, Y. M. Makeenko, J. Nishimura, and R. J. Szabo, Finite N matrix models of noncommutative gauge theory, J. High Energy Phys. 11 (1999) 029.

[19] M. R. Douglas and C. M. Hull, D-branes and the noncommutative torus, J. High Energy Phys. 02 (1998) 008.

[20] J. Madore, S. Schraml, and P. Schupp, and J. Wess, Gauge theory on noncommutative spaces, Eur. Phys. J. C 16, 161 (2000).

[21] M. R. Douglas and N. A. Nekrasov, Noncommutative field theory, Rev. Mod. Phys. 73, 977 (2001).

[22] S. Brahma, A. Marcianò, and M. Ronco, Quantum position operator: Why space-time lattice is fuzzy, arXiv:1707 .05341 .

[23] A. Connes, Noncommutative Geometry (Academic Press, New York, 1994).

[24] A. Connes and M. Marcolli, A walk in the noncommutative garden, arXiv:math/0601054.

[25] G. Landi, An Introduction to Noncommutative Spaces and Their Geometries, Lectures notes in physics (SpringerVerlag, Berlin, Heidelberg, 1997).

[26] T. Brzezinski and S. Majid, Quantum group gauge theory on quantum spaces, Commun. Math. Phys. 157, 591 (1993).

[27] M. Maggiore, Quantum groups, gravity and the generalized uncertainty principle, Phys. Rev. D 49, 5182 (1994).

[28] S. Majid, Quantum groups and noncommutative geometry, J. Math. Phys. 41, 3892 (2000).

[29] R. Akhoury and Y. P. Yao, Minimal length uncertainty relation and the hydrogen spectrum, Phys. Lett. B 572, 37 (2003).

[30] A. Agostini, G. Amelino-Camelia, and F. D'Andrea, Hopf algebra description of noncommutative space-time symmetries, Int. J. Mod. Phys. A 19, 5187 (2004).

[31] R. Oeckl, Braided quantum field theory, Commun. Math. Phys. 217, 451 (2001).

[32] L. Jonke and S. Meljanac, Representations of noncommutative quantum mechanics and symmetries, Eur. Phys. J. C 29, 433 (2003).

[33] M. Arzano and A. Marciano, Fock space, quantum fields and $\kappa$-Poincare symmetries, Phys. Rev. D 76, 125005 (2007).

[34] S. L. Woronowicz, Differential calculus on compact matrix pseudogroups (quantum groups), Commun. Math. Phys. 122, 125 (1989).
[35] X. Calmet, B. Jurco, P. Schupp, J. Wess, and M. Wohlgenannt, The standard model on noncommutative space-time, Eur. Phys. J. C 23, 363 (2002).

[36] F. Girelli, E. R. Livine, and D. Oriti, 4d deformed special relativity from group field theories, Phys. Rev. D 81, 024015 (2010).

[37] S. Ferrara, M. A. Lledo, and O. Macia, Supersymmetry in noncommutative superspaces, J. High Energy Phys. 09 (2003) 068.

[38] S. Terashima and J.-T. Yee, Comments on noncommutative superspace, J. High Energy Phys. 12 (2003) 053.

[39] M. Ihl and C. Saemann, Drinfeld-twisted supersymmetry and non-anticommutative superspace, J. High Energy Phys. 01 (2006) 065.

[40] V. G. Drinfeld, Hopf algebras and the quantum YangBaxter equation, Sov. Math. Dokl. 32, 254 (1985).

[41] V. G. Drinfeld, Quantum groups, J. Sov. Math. 41, 898 (1988).

[42] A. S. Cattaneo and G. Felder, Poisson sigma models and deformation quantization, Mod. Phys. Lett. A 16, 179 (2001).

[43] G. Amelino-Camelia, Relativity in space-times with short distance structure governed by an observer independent (Planckian) length scale, Int. J. Mod. Phys. D 11, 35 (2002).

[44] G. Amelino-Camelia, Testable scenario for relativity with minimum length, Phys. Lett. B 510, 255 (2001).

[45] L. Freidel and E. R. Livine, Effective 3-D Quantum Gravity and Non-commutative Quantum Field Theory, Phys. Rev. Lett. 96, 221301 (2006).

[46] H.-J. Matschull and M. Welling, Quantum mechanics of a point particle in $(2+1)$-dimensional gravity, Classical Quantum Gravity 15, 2981 (1998).

[47] F. A. Bais, N.M. Muller, and B. J. Schroers, Quantum group symmetry and particle scattering in $(2+1)$-dimensional quantum gravity, Nucl. Phys. B640, 3 (2002).

[48] G. Amelino-Camelia, L. Smolin, and A. Starodubtsev, Quantum symmetry, the cosmological constant and Planck scale phenomenology, Classical Quantum Gravity 21, 3095 (2004).

[49] A. Ballesteros, F. J. Herranz, and C. Meusburger, Threedimensional gravity and Drinfel'd doubles: spacetimes and symmetries from quantum deformations, Phys. Lett. B 687, 375 (2010).

[50] A. Ballesteros, F. J. Herranz, and C. Meusburger, Drinfel'd doubles for $(2+1)$-gravity, Classical Quantum Gravity 30, 155012 (2013).

[51] A. Ballesteros, F. J. Herranz, and C. Meusburger, A (2 + 1) non-commutative Drinfel'd double spacetime with cosmological constant, Phys. Lett. B 732, 201 (2014).

[52] A. Ballesteros, C. Meusburger, and P. Naranjo, AdS Poisson homogeneous spaces and Drinfel'd doubles, J. Phys. A 50, 395202 (2017).

[53] A. Ballesteros, G. Gubitosi, I. Gutiérrez-Sagredo, and F. J. Herranz, Curved momentum spaces from quantum groups with cosmological constant, Phys. Lett. B 773, 47 (2017).

[54] G. Amelino-Camelia, M. Arzano, S. Bianco, and R. J. Buonocore, The DSR-deformed relativistic symmetries 
and the relative locality of 3D quantum gravity, Classical Quantum Gravity 30, 065012 (2013).

[55] N. Seiberg and E. Witten, String theory and noncommutative geometry, J. High Energy Phys. 09 (1999) 032.

[56] E. Witten, Noncommutative geometry and string field theory, Nucl. Phys. B268, 253 (1986).

[57] E. Witten, Noncommutative tachyons and string field theory, arXiv:hep-th/0006071.

[58] N. Seiberg, L. Susskind, and N. Toumbas, Space-time noncommutativity and causality, J. High Energy Phys. 06 (2000) 044.

[59] J. de Boer, P. A. Grassi, and P. van Nieuwenhuizen, Noncommutative superspace from string theory, Phys. Lett. B 574, 98 (2003).

[60] A. Sitarz, Noncommutative differential calculus on the $\kappa$-Minkowski space, Phys. Lett. B 349, 42 (1995).

[61] S. Meljanac, A. Samsarov, M. Stojic, and K. S. Gupta, $\kappa$-Minkowski space-time and the star product realizations, Eur. Phys. J. C 53, 295 (2008).

[62] P. Podles and S. L. Woronowicz, Quantum deformation of Lorentz group, Commun. Math. Phys. 130, 381 (1990).

[63] V. Gayral, J. M. Gracia-Bondia, B. Iochum, T. Schucker, and J.C. Varilly, Moyal planes are spectral triples, Commun. Math. Phys. 246, 569 (2004).

[64] G. Amelino-Camelia, Quantum-spacetime phenomenology, Living Rev. Relativity 16, 5 (2013).

[65] H. S. Snyder, Quantized space-time, Phys. Rev. 71, 38 (1947).

[66] V. Schomerus, D-branes and deformation quantization, J. High Energy Phys. 06 (1999) 030.

[67] S. Majid and H. Ruegg, Bicrossproduct structure of $\kappa$-Poincare group and noncommutative geometry, Phys. Lett. B 334, 348 (1994).

[68] J. Lukiersk, A. Nowicki, and H. Ruegg, New quantum Poincare algebra and $\mathrm{k}$ deformed field theory, Phys. Lett. B 293, 344 (1992).

[69] J. Lukierski, H. Ruegg, A. Nowicki, and V. N. Tolstoi, Q deformation of Poincare algebra, Phys. Lett. B 264, 331 (1991).

[70] S. Majid, Braided momentum in the Q Poincare group, J. Math. Phys. 34, 2045 (1993).

[71] S. Giller, P. Kosinski, M. Majewski, P. Maslanka, and J. Kunz, More about Q deformed Poincare algebra, Phys. Lett. B 286, 57 (1992).

[72] A. P. Balachandran, S. Kurkcuoglu, and E. Rojas, The star product on the fuzzy supersphere, J. High Energy Phys. 07 (2002) 056.

[73] A. Marciano, G. Amelino-Camelia, N. R. Bruno, G. Gubitosi, G. Mandanici, and A. Melchiorri, Interplay between curvature and Planck-scale effects in astrophysics and cosmology, J. Cosmol. Astropart. Phys. 06 (2010) 030.

[74] A. Ballesteros, N. R. Bruno, and F. J. Herranz, A noncommutative Minkowskian space-time from a quantum AdS algebra, Phys. Lett. B 574, 276 (2003).

[75] G. Amelino-Camelia, A. Marciano, M. Matassa, and G. Rosati, Deformed Lorentz symmetry and relative locality in a curved/expanding spacetime, Phys. Rev. D 86, 124035 (2012).

[76] X. Calmet and A. Kobakhidze, Noncommutative general relativity, Phys. Rev. D 72, 045010 (2005).
[77] L. Smolin, The quantization of unimodular gravity and the cosmological constant problems, Phys. Rev. D 80, 084003 (2009).

[78] E. Álvarez, S. González-Martín, M. Herrero-Valea, and C. P. Martín, Quantum corrections to unimodular gravity, J. High Energy Phys. 08 (2015) 078.

[79] A. H. Chamseddine, G. Felder, and J. Frohlich, Gravity in noncommutative geometry, Commun. Math. Phys. 155, 205 (1993).

[80] H. Steinacker, Emergent gravity from noncommutative gauge theory, J. High Energy Phys. 12 (2007) 049.

[81] H. Steinacker, Emergent geometry and gravity from matrix models: An introduction, Classical Quantum Gravity 27, 133001 (2010).

[82] J. Madore, The commutative limit of a matrix geometry, J. Math. Phys. 32, 332 (1991).

[83] H. Grosse and J. Madore, A noncommutative version of the Schwinger model, Phys. Lett. B 283, 218 (1992).

[84] A. P. Balachandran, S. Kurkcuoglu, and S. Vaidya, Lectures on Fuzzy and Fuzzy SUSY Physics (World Scientific, Singapore, 2007), p. 191.

[85] H. Steinacker, Emergent gravity on covariant quantum spaces in the IKKT model, J. High Energy Phys. 12 (2016) 156.

[86] M. Sperling and H. Steinacker, Higher spin gauge theory on fuzzy $S_{N}^{4}$, J. Phys. A 51, 075201 (2018).

[87] P. Aschieri, C. Blohmann, M. Dimitrijevic, F. Meyer, P. Schupp, and J. Wess, A gravity theory on noncommutative spaces, Classical Quantum Gravity 22, 3511 (2005).

[88] P. Aschieri, M. Dimitrijevic, F. Meyer, and J. Wess, Noncommutative geometry and gravity, Classical Quantum Gravity 23, 1883 (2006).

[89] L. Alvarez-Gaume, F. Meyer, and M. A. Vazquez-Mozo, Comments on noncommutative gravity, Nucl. Phys. B753, 92 (2006).

[90] A. Duenas-Vidal and M. A. Vazquez-Mozo, Twisted invariances of noncommutative gauge theories, Phys. Lett. B 668, 57 (2008).

[91] P. A. M. Dirac, The theory of gravitation in Hamiltonian form, Proc. R. Soc. A 246, 333 (1958).

[92] R. L. Arnowitt, S. Deser, and C. W. Misner, The dynamics of general relativity, Gen. Relativ. Gravit. 40, 1997 (2008).

[93] T. Regge and C. Teitelboim, Role of surface integrals in the Hamiltonian formulation of general relativity, Ann. Phys. (N.Y.) 88, 286 (1974).

[94] M. Bojowald, Canonical Gravity and Applications: Cosmology, Black Holes, and Quantum Gravity (Cambridge University Press, Cambridge, England, 2010).

[95] C. Blohmann, M. C. B. Fernandes, and A. Weinstein, Groupoid symmetry and constraints in general relativity, Commun. Contemp. Math. 15, 1250061 (2013).

[96] M. Bojowald, S. Brahma, U. Buyukcam, and F. D' Ambrosio, Hypersurface-deformation algebroids and effective spacetime models, Phys. Rev. D 94, 104032 (2016).

[97] S. Majid and R. Oeckl, Twisting of quantum differentials and the Planck scale Hopf algebra, Commun. Math. Phys. 205, 617 (1999).

[98] E. Langmann, R. J. Szabo, and K. Zarembo, Exact solution of quantum field theory on noncommutative phase spaces, J. High Energy Phys. 01 (2004) 017. 
[99] R. Britto, B. Feng, and S.-J. Rey, Non(anti)commutative superspace, UV / IR mixing and open Wilson lines, J. High Energy Phys. 08 (2003) 001.

[100] S. Ferrara, E. Ivanov, O. Lechtenfeld, E. Sokatchev, and B. Zupnik, Non-anticommutative chiral singlet deformation of $\mathrm{N}=(1,1)$ gauge theory, Nucl. Phys. B704, 154 (2005).

[101] A. Kempf, On noncommutative geometric regularization, Phys. Rev. D 54, 5174 (1996); Erratum, Phys. Rev. D 55, 1114(E) (1997).

[102] L. Freidel, R. G. Leigh, and D. Minic, Intrinsic noncommutativity of closed string theory, arXiv:1706.03305.

[103] L. Freidel, R. G. Leigh, and D. Minic, Noncommutativity of closed string zero modes, Phys. Rev. D 96, 066003 (2017).

[104] L. Freidel, R. G. Leigh, and D. Minic, Metastring theory and modular space-time, J. High Energy Phys. 06 (2015) 006.

[105] M. Bojowald and G. M. Paily, Deformed general relativity and effective actions from loop quantum gravity, Phys. Rev. D 86, 104018 (2012).

[106] M. Bojowald, S. Brahma, and J. D. Reyes, Covariance in models of loop quantum gravity: Spherical symmetry, Phys. Rev. D 92, 045043 (2015).

[107] M. Bojowald and S. Brahma, Covariance in models of loop quantum gravity: Gowdy systems, Phys. Rev. D 92, 065002 (2015).

[108] A. Perez and D. Pranzetti, On the regularization of the constraints algebra of quantum gravity in $2+1$ dimensions with non-vanishing cosmological constant, Classical Quantum Gravity 27, 145009 (2010).

[109] A. Barrau, M. Bojowald, G. Calcagni, J. Grain, and M. Kagan, Anomaly-free cosmological perturbations in effective canonical quantum gravity, J. Cosmol. Astropart. Phys. 05 (2015) 051.

[110] C. Tomlin and M. Varadarajan, Towards an anomaly-free quantum dynamics for a weak coupling limit of Euclidean gravity, Phys. Rev. D 87, 044039 (2013).

[111] A. Henderson, A. Laddha, and C. Tomlin, Constraint algebra in loop quantum gravity reloaded. I. Toy model of a $U(1)^{3}$ gauge theory, Phys. Rev. D 88, 044028 (2013).
[112] G. Calcagni and M. Ronco, Deformed symmetries in noncommutative and multifractional spacetimes, Phys. Rev. D 95, 045001 (2017).

[113] G. Calcagni, Multifractional theories: An unconventional review, J. High Energy Phys. 03 (2017) 138.

[114] M. Bojowald and G. M. Paily, Deformed general relativity, Phys. Rev. D 87, 044044 (2013).

[115] G. Amelino-Camelia, M. M. da Silva, M. Ronco, L. Cesarini, and O. M. Lecian, Spacetime-noncommutativity regime of loop quantum gravity, Phys. Rev. D 95, 024028 (2017).

[116] F. Cianfrani, J. Kowalski-Glikman, D. Pranzetti, and G. Rosati, Symmetries of quantum spacetime in three dimensions, Phys. Rev. D 94, 084044 (2016).

[117] G. Amelino-Camelia, M. Arzano, M. M. Da Silva, and D. H. Orozco-Borunda, Relativistic Planck-scale polymer, arXiv:1707.05017.

[118] S. Brahma, M. Ronco, G. Amelino-Camelia, and A. Marciano, Linking loop quantum gravity quantization ambiguities with phenomenology, Phys. Rev. D 95, 044005 (2017).

[119] J. Mielczarek, Loop-deformed Poincare algebra, Europhys. Lett. 108, 40003 (2014).

[120] M. Ronco, On the UV dimensions of loop quantum gravity, Adv. High Energy Phys. 2016, 1 (2016).

[121] J. Mielczarek and T. Trześniewski, Spectral dimension with deformed spacetime signature, Phys. Rev. D 96, 024012 (2017).

[122] H. Gomes and V. Shyam, Extending the rigidity of general relativity, J. Math. Phys. (N.Y.) 57, 112503 (2016).

[123] P. Xu, Quantum groupoids, Commun. Math. Phys. 216, 539 (2001).

[124] J. E. Moyal, Quantum mechanics as a statistical theory, Proc. Cambridge Philos. Soc. 45, 99 (1949).

[125] P. Schupp, Quantum groups, noncommutative differential geometry and applications, arXiv:hep-th/9312075.

[126] F. Koch and E. Tsouchnika, Construction of $\theta$-Poincare algebras and their invariants on $\mathcal{M}_{\theta}$, Nucl. Phys. B717, 387 (2005). 